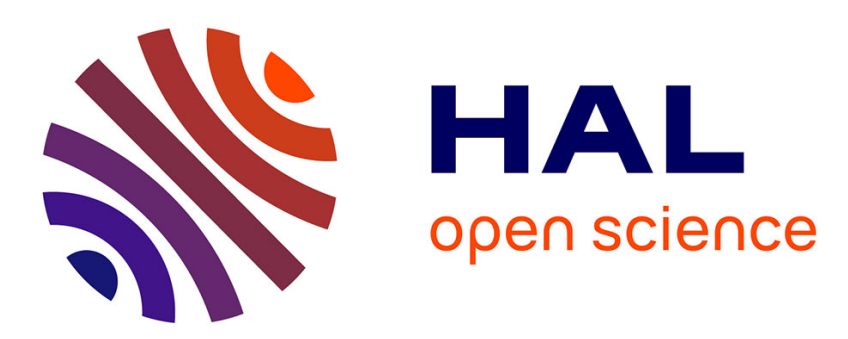

\title{
A hybrid transport-diffusion model for radiative transfer in absorbing and scattering media
}

Maxime Roger, Cyril Caliot, Nicolas Crouseilles, Pedro Jorge Martins Coelho

\section{To cite this version:}

Maxime Roger, Cyril Caliot, Nicolas Crouseilles, Pedro Jorge Martins Coelho. A hybrid transportdiffusion model for radiative transfer in absorbing and scattering media. Journal of Computational Physics, 2014, 275, pp.346 - 362. 10.1016/j.jcp.2014.06.063 . hal-01090680

\section{HAL Id: hal-01090680 \\ https://hal.inria.fr/hal-01090680}

Submitted on 4 Dec 2014

HAL is a multi-disciplinary open access archive for the deposit and dissemination of scientific research documents, whether they are published or not. The documents may come from teaching and research institutions in France or abroad, or from public or private research centers.
L'archive ouverte pluridisciplinaire HAL, est destinée au dépôt et à la diffusion de documents scientifiques de niveau recherche, publiés ou non, émanant des établissements d'enseignement et de recherche français ou étrangers, des laboratoires publics ou privés. 


\title{
A hybrid transport-diffusion model for radiative transfer in absorbing and scattering media
}

\author{
M. Roger ${ }^{\mathrm{a}}$, C. Caliot $^{\mathrm{b}}$, N. Crouseilles ${ }^{\mathrm{c}}$, P.J. Coelho ${ }^{\mathrm{d}}$ \\ ${ }^{a}$ Université de Lyon, CNRS, CETHIL UMR5008, F-69621, France \\ ${ }^{b}$ PROMES-UPR CNRS 6144, 7 rue du Four Solaire, 66120 Font Romeu Odeillo, France \\ ${ }^{c}$ INRIA-Rennes Bretagne-Atlantique (IPSO Project) and Université de Rennes 1 (IRMAR), Campus \\ de Beaulieu, 35042 Rennes Cedex, France \\ ${ }^{d}$ Mechanical Engineering Department, Instituto Superior Técnico/IDMEC, University of Lisbon, Av. \\ Rovisco Pais, 1049-001 Lisboa, Portugal
}

\begin{abstract}
A new multi-scale hybrid transport-diffusion model for radiative transfer calculations is proposed. In this model, the radiative intensity is decomposed into a macroscopic component calculated by the diffusion equation, and a mesoscopic component. The transport equation for the mesoscopic component allows to correct the estimation of the diffusion equation, and then to obtain the solution of the linear radiative transfer equation. In this work, results are presented for stationary and transient radiative transfer cases, in examples which concern solar concentrated and optical tomography applications. The Monte Carlo and the discrete-ordinate methods are used to solve the mesoscopic equation. It is shown that the multi-scale model allows to improve the efficiency of the calculations when the medium is close to the diffusive regime. Moreover, the development of methods for coupling the radiative transfer equation with the diffusion equation becomes easier with this model than with the usual domain decomposition methods.

Keywords:

Radiative transfer, multi-scale model, hybrid transport-diffusion model, Monte Carlo method, Discrete ordinates method
\end{abstract}

\section{Introduction}

Linear transport models are applied in many research fields including neutron transport [1], thermal radiation [2], medical imaging [3] or atmospheric physics [4], among others. In this work, we focus on radiative transfer models in absorbing and scat- 
tering media for applications such as solar energy processes and optical tomography. Actually, two principal models are generally used for linear radiation transport : the radiative transfer equation (RTE) defined at the kinetic scale and the macroscopic diffusion equation (DE).

The RTE is a mesoscopic model which offers a better accuracy than the DE but requires a higher computational effort. Several numerical methods such as the Monte Carlo methods (MCM), the discrete-ordinates (DOM) or the finite-volume methods (FVM) have been developed over the last decades in order to solve the RTE with precision and efficiency. The DOM and the FVM are deterministic methods, frequently applied in engineering problems, which are generally faster than the MCM [2]. However, with the increase of computational power, the MCM become more attractive and has been used in various engineering applications [5]. Accordingly, the Monte Carlo algorithms have been significantly improved in concentrated solar processes [6], remote sensing [7] or for sensitivity estimations [8].

All these methods based on the RTE converge slowly when the medium is close to the diffusive regime. Accordingly, the computational requirements increase significantly with the scattering optical thickness. In the case of MCM, the CPU time increases with the number of times that photons scatter during their optical path, and the statistical error improves as well. In the case of DOM or FVM, the numerical parameters must respect strong constraints for stability reasons (like the mesh size which has to be lower than the mean free path, very small if the scattering optical thickness is large). At the diffusive regime, the radiation model which is generally used is the DE if it is assumed that the radiative intensity is almost isotropic (P1 approximation), and that the radiative heat flux scales with the gradient of the incident radiation [2]. The DE is much more easier to solve than the RTE, and is therefore an efficient model in highly scattering media. But the DE has some major drawbacks in the proximity of boundaries or sources, or in regions where the medium is not optically thick, where the $\mathrm{P} 1$ approximation is not valid anymore.

The objective of this work is to propose a radiation model for multi-scale problems where one has to deal with diffusive and kinetic regimes. We will focus on a first test case related to radiation transport in a volumetric receiver in concentrated 
solar applications. The solar concentrated processes involve a concentrating system (heliostat field) and a receiver. High temperature volumetric solar receivers have been a research topic for more than 30 years [9]. The aim of volumetric receivers is to use semi-transparent media (particles, wire meshes, fibers, honeycombs, reticulated porous ceramics) to absorb solar radiation deep inside the absorber in order to decrease losses by reflections and thermal emission. Recent studies $[10,11]$ used the DE for the predicition of thermal radiation inside a volumetric solar absorber. The DE leads to acceptable computation time, but it cannot estimate accurately the losses at the inlet boundary. In these applications, a radiation multi-scale model, which is able to predict correctly the losses by reflections or emission close to the surface irradiated by the concentrated solar fluxes, and to predict correctly radiative transfer inside the volumetric absorber where the medium is close to the diffusive regime, is needed.

On a second time, an academic problem related to optical tomography applications will be studied. The propagation of a short-pulse laser into a biological tissue allows to estimate the optical properties of the tissue and to detect inhomogeneities and tumors $[12,13]$. In biological tissue, the medium is generally optically thick, and scattering dominates over absorption, which means that the DE can be assumed for radiation model [14]. However, the diffusion approximation has some limitations at the boundaries of the system where the short-pulse laser is emitted. The collimated irradiation cannot be simulated accurately with the DE, and needs a kinetic description. Moreover, scattering is typically forward-peaked in tissues [15], which enhances the need for a mesoscopic model close to the boundaries. in such cases, a transient multiscale radiation model is needed to simulate the propagation of the short-pulse laser inside the tissue.

In multi-scale problems, a solution is to couple the DE with the RTE using a domain decomposition method, in which the system is decomposed into a mesoscopic subdomain where the RTE is solved and a macroscopic subdomain where the DE is solved. This approach has been tested in various studies $[16,17,18,15]$. In these works, the treatment of the geometric interface between the macroscopic and the mesoscopic subdomains represents the major difficulty to handle [19]. The boundary conditions at this interface for the DE must be consistent with the boundary conditions for the 
RTE, which is not an easy task and can lead to strong error [15]. In our previous work, a dynamic multi-scale model based on the domain-decomposition strategy has been proposed [20] which allows to overcome the interface treatment difficulties. In this model, the DE and the RTE are coupled through the equations instead of being coupled through a geometric interface. A buffer zone is introduced between the kinetic and the diffusive subdomain, and the coupling is dealt inside this buffer zone. Note that no boundary conditions are needed for the DE with this model.

Another possible approach for dealing with multi-scale problems is based on the so-called micro-macro formulation [21, 22, 23]. In this formulation, the mesoscopic unknown is split into a mesoscopic and a macroscopic components, and a two-way coupled system of equations is obtained for these two components. The micro-macro model is equivalent to the initial kinetic equation, unlike domain decomposition methods. During the derivation of the macroscopic equation from the kinetic equation, the exact boundary conditions are generally lost, and artificial boundary conditions may be needed. However, it is possible to find an alternative decomposition which matches the exact boundary conditions as proposed in [24]. The principal problem of the micromacro formulation is to deal with the coupling between the macroscopic and the kinetic equations; indeed, the mesoscopic equation cannot be solved easily and efficiently with a Monte Carlo method because of the coupling with the macroscopic equation.

In this work, a new multi-scale hybrid transport-diffusion (HTD) model is proposed for radiative transfer. In this model, similarly to the micro-macro model, the radiative intensity is decomposed into a macroscopic and a mesoscopic components, leading to a macroscopic and a mesoscopic equations. The major difference with the micro-macro model is that the two equations are one-way coupled instead of being two-way coupled. The macroscopic equation does not depend on the resolution of the mesoscopic equation and can be solved independently. Therefore, the macroscopic diffusion equation is first solved on the whole time and space intervals, and then the mesoscopic equation is solved with a source term depending on the unknown of the macroscopic equation previously computed. The model obtained enables to reconstruct the solution of the RTE by adding the solution of the diffusion equation and the solution of the mesoscopic equation. In particular, the exact boundary conditions are conserved. 
In the next section, the models for radiative transfer are presented. In section 3 , the HTD model is tested with the MCM in a stationary test case related to concentrated solar applications. A transient test case related to optical tomography application is studied in section 4 with the DOM and the MCM.

\section{Radiative transfer models}

\subsection{The RTE and the DE}

The transient RTE in absorbing, emitting and scattering media can be written as

$$
\frac{1}{c} \frac{\partial I}{\partial t}+\boldsymbol{u} \cdot \nabla I=-\left(k_{a}+k_{s}\right) I+k_{a} I_{b}+k_{s} \int_{4 \pi} p\left(\boldsymbol{u} \mid \boldsymbol{u}^{\prime}\right) I^{\prime} d \boldsymbol{u}^{\prime}
$$

where $I=I(\boldsymbol{x}, \boldsymbol{u}, t)$ is the radiative intensity at location $\boldsymbol{x}$, propagation direction $\boldsymbol{u}$ and time $t$. The radiative intensity $I$ is linked to the photons distribution function $f$ according to $I=h \nu c f$, where $h$ is the Planck constant, $\nu$ the frequency of the photons and $c$ the speed of light. The other quantities of Eq. 1 are the following: $k_{a}(\boldsymbol{x}, t)$ is the absorption coefficient, $k_{s}(\boldsymbol{x}, t)$ the scattering coefficient, $p\left(\boldsymbol{x}, \boldsymbol{u} \mid \boldsymbol{u}^{\prime}\right)$ is the normalized scattering phase function and $\boldsymbol{u}^{\prime}$ is the incident direction. Finally $I_{b}(\boldsymbol{x}, t)$ denotes the Planck function and the notation $I^{\prime}$ stands for $I^{\prime} \equiv I\left(\boldsymbol{x}, \boldsymbol{u}^{\prime}, t\right)$.

The RTE integrated over the solid-angle space can be written as:

$$
\frac{1}{c} \frac{\partial \rho}{\partial t}+\nabla \cdot j=k_{a}\left(4 \pi I_{b}-\rho\right)
$$

In this equation, $\rho$ is the photon fluence rate (or incident radiation), defined by $\rho(\boldsymbol{x}, t)=$ $\langle I(\boldsymbol{x}, \boldsymbol{u}, t)\rangle$, where the symbol $\langle\cdot\rangle$ represents the integral over the solid-angle space $\left(\langle\alpha\rangle=\int_{4 \pi} \alpha(\boldsymbol{u}) d \boldsymbol{u}\right)$. Let us remark that $\rho$ can be linked to the photon density $\Phi$ according to $\rho=h \nu c \Phi$. Finally, $\boldsymbol{j}$ is the radiative heat flux defined by $\boldsymbol{j}(\boldsymbol{x}, t)=$ $\langle I(\boldsymbol{x}, \boldsymbol{u}, t) \boldsymbol{u}\rangle$.

Eq. 2 is not closed and an assumption has to be done to express the radiative heat flux $\boldsymbol{j}$ in term of the fluence rate $\rho$. If the $P 1$ approximation is assumed for the intensity $I^{l i m}=\frac{\rho^{l i m}}{4 \pi}+\frac{3}{4 \pi}\left(\boldsymbol{j}^{l i m} \cdot \boldsymbol{u}\right)$, as well as the diffusion approximation $\frac{\partial \boldsymbol{j}^{l i m}}{\partial t}=0$ [25], the radiative heat flux at the diffusive limit $\boldsymbol{j}^{l i m}=\left\langle I^{l i m} \cdot \boldsymbol{u}\right\rangle$ is related to $\rho^{l i m}$ according to

$$
\boldsymbol{j}^{l i m}=-D \nabla \rho^{l i m}, \text { with } D=\frac{1}{3\left[k_{a}+k_{s}(1-g)\right]},
$$


where $D$ is the diffusion coefficient and $g$ is the anisotropy factor defined by $g=$ $\int_{0}^{1} p(\mu) \mu d \mu$ with $\mu=\boldsymbol{u} \cdot \boldsymbol{u}^{\prime}$. In Eq. 3, the phase function is assumed to depend only on the scalar product between the incident and the scattered direction $p\left(\boldsymbol{u} \mid \boldsymbol{u}^{\prime}\right)=p\left(\boldsymbol{u} \cdot \boldsymbol{u}^{\prime}\right)$, which is not a restrictive assumption in the applications of interest. The macroscopic diffusion equation is then deduced from Eqs. 2 and 3:

$$
\frac{1}{c} \frac{\partial \rho^{l i m}}{\partial t}-\nabla \cdot\left[D \nabla \rho^{l i m}\right]=k_{a}\left(4 \pi I_{b}-\rho^{l i m}\right)
$$

\subsection{Hybrid transport-diffusion model for radiative transfer}

The hybrid transport-diffusion (HTD) model decomposes the radiative intensity according to

$$
I(\boldsymbol{x}, \boldsymbol{u}, t)=\frac{\rho^{l i m}(\boldsymbol{x}, t)}{4 \pi}+\epsilon(\boldsymbol{x}, \boldsymbol{u}, t)
$$

The macroscopic unknown is the photon fluence rate at the diffusive limit, $\rho^{\text {lim }}$, which satisfies the DE (Eq. 4). The transport equation for the mesoscopic component $\epsilon$ is then deduced from the RTE (Eq. 1) and from the DE,

$$
\frac{1}{c} \frac{\partial \epsilon}{\partial t}+\boldsymbol{u} \cdot \nabla \epsilon=-\left(k_{a}+k_{s}\right) \epsilon+k_{s} \int_{4 \pi} p\left(\boldsymbol{u} \cdot \boldsymbol{u}^{\prime}\right) \epsilon^{\prime} d \boldsymbol{u}^{\prime}+S_{\rho^{l i m}}
$$

The source term which depends on $\rho^{\text {lim }}$ in Eq. 6 can be written as:

$$
S_{\rho^{l i m}}=\frac{1}{4 \pi}\left[\nabla \cdot \boldsymbol{j}^{l i m}-\boldsymbol{u} \cdot \nabla \rho^{l i m}\right]
$$

where $\boldsymbol{j}^{\text {lim }}$ is given by Eq. 3. $S_{\rho^{l i m}}$ can be either positive or negative, as well as $\epsilon$. When the DE solution $\frac{\rho^{\lim (\boldsymbol{x}, t)}}{4 \pi}$ overpredicts the radiation intensity $I(\boldsymbol{x}, \boldsymbol{u}, t), \epsilon(\boldsymbol{x}, \boldsymbol{u}, t)$ becomes negative to compensate this effect, according to Eq. 5. Note that Eq. 6 is very similar to the RTE, and therefore the usual methods developed for solving the RTE can be easily adapted to solve the transport equation for $\epsilon$. The exact boundary conditions $(\mathrm{BC})$ on $I$ are conserved with the HTD model. Indeed, the BC for $\epsilon$ are defined according to the chosen boundary condition on $\rho^{\text {lim }}$, in order to match the exact intensity $I\left(\boldsymbol{x}_{\boldsymbol{w}}, \boldsymbol{u}, t\right)$, such as:

$$
\epsilon\left(\boldsymbol{x}_{\boldsymbol{w}}, \boldsymbol{u}, t\right)=I\left(\boldsymbol{x}_{\boldsymbol{w}}, \boldsymbol{u}, t\right)-\frac{\rho^{l i m}\left(\boldsymbol{x}_{\boldsymbol{w}}, t\right)}{4 \pi} \text { for } \boldsymbol{u} \cdot \boldsymbol{n}>0
$$


where $\boldsymbol{x}_{\boldsymbol{w}}$ is the boundary point and $\boldsymbol{n}$ is the ingoing normal at position $\boldsymbol{x}_{\boldsymbol{w}}$. Note that in the remainder of this article, the Marshak boundary conditions are used for $\rho^{\lim }\left(\boldsymbol{x}_{\boldsymbol{w}}, t\right):$

$$
\rho^{l i m}\left(\boldsymbol{x}_{\boldsymbol{w}}, t\right)+2 \boldsymbol{j}^{l i m}\left(\boldsymbol{x}_{\boldsymbol{w}}, t\right) \cdot \boldsymbol{n}=4 \int_{\boldsymbol{u} \cdot \boldsymbol{n}>0} I\left(\boldsymbol{x}_{\boldsymbol{w}}, \boldsymbol{u}, t\right)(\boldsymbol{u} \cdot \boldsymbol{n}) d \boldsymbol{u} .
$$

The procedure to solve the HTD model is straightforward:

- Solve the DE (Eq. 3) in order to calculate the field of $\rho^{l i m}$ in the system and for the considered time interval.

- Solve the transport equation on $\epsilon$ (Eq. 6) by using one of the usual methods to solve the RTE, such as the MCM, the DOM or the FVM, among others.

- Estimate the radiation quantity by adding the solution of both equations. For instance if the fluence rate $\rho$ is needed: $\rho=\rho^{l i m}+\rho_{\epsilon}$ where $\rho_{\epsilon}=\langle\epsilon\rangle$.

In the micro-macro model proposed in $[22,23]$, the mesoscopic distribution function is also decomposed into a macroscopic and a mesoscopic component. The major difference with the HTD model is that the two equations obtained are two-way coupled, and consequently less flexible to solve.

\subsection{Illustrative multi-dimensional test case in the stationary regime with the Monte}

\section{Carlo method}

In the stationary regime, the DE is simplified to

$$
-\nabla \cdot\left[D \nabla \rho^{l i m}\right]=k_{a}\left(4 \pi I_{b}-\rho^{l i m}\right) .
$$

Eq. 10 is also called the P1 model, because in stationary cases, only the P1 approxima-

tion is needed to obtain the macroscopic model (the diffusion approximation $\frac{\partial j^{l i m}}{\partial t}=0$ is always verified). The transport equation for $\epsilon$ in the HTD model becomes

$$
\boldsymbol{u} \cdot \nabla \epsilon=-\left(k_{a}+k_{s}\right) \epsilon+k_{s} \int_{4 \pi} p\left(\boldsymbol{u} \cdot \boldsymbol{u}^{\prime}\right) \epsilon^{\prime} d \boldsymbol{u}^{\prime}+S_{\rho^{l i m}}
$$

where $S_{\rho^{l i m}}$ can be written as

$$
S_{\rho^{l i m}}=k_{a}\left(I_{b}-\frac{\rho^{l i m}}{4 \pi}\right)-\frac{\boldsymbol{u} \cdot \nabla \rho^{l i m}}{4 \pi}
$$


The illustrative example considered is a homogeneous absorbing and isotropic scattering three-dimensional cubic enclosure, filled with a uniform grey gas at temperature $1000 K$ confined between black walls at $300 K$. The length of the cube is $1 m$ and the number of grid meshes is $15^{3}$.

In a first step, the P1 model (Eq. 10) is solved to get $\rho^{\text {lim }}$ by a classical second order finite volume method. The finite volume discretization leads to a linear matrix system which is solved by the iterative Gauss-Seidel method.

Once the field of $\rho^{l i m}$ is known, a MC algorithm based on the integral formulation is developed for the estimation of $\rho_{\epsilon}=\langle\epsilon\rangle$ (in order to calculate $\rho=\rho^{\lim }+\rho_{\epsilon}$ ). In Appendix A, a classical reverse MC algorithm for the estimation of $\rho$ is detailed. It is based on the following formulation:

$$
\rho(\boldsymbol{x})=\int_{4 \pi} p_{\boldsymbol{U}}(\boldsymbol{u}) d \boldsymbol{u} \int_{0}^{+\infty} p_{L}\left(\ell_{1}\right) d \ell_{1} \int_{4 \pi} p_{\boldsymbol{U}_{\mathbf{1}}}\left(\boldsymbol{u}_{\mathbf{1}}\right) d \boldsymbol{u}_{\mathbf{1}} \times w
$$

where $w$ is the Monte Carlo weight expressed by

$$
w=4 \pi\left\{H\left(\ell_{1}-d_{1}\right) I\left(\boldsymbol{x}_{\boldsymbol{w} \mathbf{1}}, \boldsymbol{u}\right)+H\left(d_{1}-\ell_{1}\right)\left\{\left(1-\omega\left(\boldsymbol{x}_{\mathbf{1}}\right)\right) I_{b}\left(\boldsymbol{x}_{\mathbf{1}}\right)+\omega\left(\boldsymbol{x}_{\mathbf{1}}\right) I\left(\boldsymbol{x}_{\mathbf{1}}, \boldsymbol{u}_{\mathbf{1}}\right)\right\}\right\}
$$

where $\omega=\frac{k_{s}}{k_{a}+k_{s}}$ is the scattering albedo, $H(x)$ is the Heaviside function defined as $H(x)=1$ if $x \geq 0$, and $H(x)=0$ if $x<0, \ell_{1}=\left\|\boldsymbol{x}-\boldsymbol{x}_{\mathbf{1}}\right\|$ and $d_{1}=\left\|\boldsymbol{x}-\boldsymbol{x}_{\boldsymbol{w} \mathbf{1}}\right\|$. In figure 1 the notations are illustrated in an example of optical path. The chosen probability density functions (pdf) are:

$$
\begin{aligned}
& p_{\boldsymbol{U}}(\boldsymbol{u})=\frac{1}{4 \pi} \\
& p_{L}\left(\ell_{i}\right)=\left(k_{a}+k_{s}\right) \exp \left[-\int_{0}^{\ell_{i}}\left(k_{a}+k_{s}\right) d \ell\right], \quad \forall i \in \mathbb{N}^{+} \text {where } \ell_{i}=|| \boldsymbol{x}_{\boldsymbol{i}}-\boldsymbol{x}_{\boldsymbol{i - 1}} \mid(16) \\
& p_{\boldsymbol{U}_{\boldsymbol{i}}}\left(\boldsymbol{u}_{\boldsymbol{i}}\right)=p\left(\boldsymbol{u}_{\boldsymbol{i - 1}} \mid \boldsymbol{u}_{\boldsymbol{i}}\right), \quad \forall i \in \mathbb{N}^{+}
\end{aligned}
$$

In this algorithm, the optical path is randomly generated in a reverse way. At each collision position generated according to $p_{L}$, the emission source term is implemented.

The MC algorithm used to solve Eq. 11 in the HTD model is almost identical to the algorithm used to solve the RTE. The integral expression of $\rho_{\epsilon}$ is very similar to Eq. 13,

$$
\rho_{\epsilon}(\boldsymbol{x})=\int_{4 \pi} p_{\boldsymbol{u}}(\boldsymbol{u}) d \boldsymbol{u} \int_{0}^{+\infty} p_{L}\left(\ell_{1}\right) d \ell_{1} \int_{4 \pi} p_{\boldsymbol{U}_{\mathbf{1}}}\left(\boldsymbol{u}_{\mathbf{1}}\right) d \boldsymbol{u}_{\mathbf{1}} \times w_{\epsilon}
$$


where the probability density functions are unchanged and the MC weight $w_{\epsilon}$ is expressed by:

$\left.w_{\epsilon}=4 \pi\left\{H\left(\ell_{1}-d_{1}\right) \epsilon\left(\boldsymbol{x}_{\boldsymbol{w} \mathbf{1}}, \boldsymbol{u}\right)+H\left(d_{1}-\ell_{1}\right)\left\{\frac{S_{\rho^{l i m}}\left(\boldsymbol{x}_{\mathbf{1}}\right)}{k_{a}\left(\boldsymbol{x}_{\mathbf{1}}\right)+k_{s}\left(\boldsymbol{x}_{\mathbf{1}}\right)}+\omega\left(\boldsymbol{x}_{\mathbf{1}}\right) \epsilon\left(\boldsymbol{x}_{\mathbf{1}}, \boldsymbol{u}_{\mathbf{1}}\right)\right\} 1\right\}\right)$

where $S_{\rho^{l i m}}$ is given by Eq. 12. When comparing the expression of the MC weight $w$ (Eq. 14) with the expression of $w_{\epsilon}$ (Eq. 19), it is clear that the algorithms are very similar. The only difference is that $w_{\epsilon}$ is now implemented by the source term $S_{\rho^{l i m}}$ from Eq. 12 at each scattering position, instead of being implemented by the emission. The value of $\rho^{\text {lim }}$ and of its gradient at the scattering position $\boldsymbol{x}_{\boldsymbol{j}}$, needed for the calculation of $S_{\rho^{\text {lim }}}\left(\boldsymbol{x}_{\boldsymbol{j}}\right)$, are obtained by linear interpolation from the values at the surrounding mesh points of the grid used to solve the DE (Eq. 10).

In figure 2, an example of MC calculations of the RTE and the HTD model is presented along with the solution of the P1 model. In this case, the optical thickness is 10 , the albedo 0.5 (which means that the absorption and the scattering coefficients are equal to 5) and the phase function is isotropic. In figure 2(a), the fluence rate along the $x$-coordinate of the enclosure is displayed ( $y$ and $z$ are fixed to 0.6). The MC solutions for the RTE and the HTD model are merged as expected, and it can be seen that the P1 model represents a good alternative to the mesoscopic models in this example, except close to the boundaries. In figure 2(b), the relative statistical uncertainties are presented. The number of optical path generated for each points of the mesh is 100000 in the case of the RTE calculations, while in the HTD calculations, this number is 50000. It is shown that the HTD model gives better results than the RTE (except at the boundary where the uncertainties are equivalent), for a lower computational time $t_{H T D}=0.6 t_{R T E}$. In this case, the MCM based on the HTD model estimates the difference between the P1 solution and the exact solution $\left(\rho-\rho^{\text {lim }}\right)$, instead of estimating directly the exact solution $\rho$ (like the MCM based on the RTE). Consequently, the statistical error with the HTD model is smaller because the quantity estimated with the HTD is smaller than with the RTE $\left(\rho-\rho^{l i m}<<\rho\right)$.

In table 1 , the ratio of the computational time needed for solving the RTE and the HTD model at equivalent accuracy are given for various optical thicknesses. When the optical thickness is 1 or thinner, the predictions of the P1 model are not accurate and 
consequently the control variate method does not ensure a better performance than the usual RTE. However, when the optical thickness increases (for $\tau \geq 5$ ), the efficiency of the HTD calculations are better than the RTE.

\section{Example of a receiver in solar concentrated applications}

\subsection{Stationary radiative transfer with collimated irradiation}

In this section, the HTD model is applied to the simulation of radiative transfer into a solar receiver irradiated by concentrated solar fluxes. The incident concentrated radiation was modelled as a collimated external source and an effective grey semitransparent medium was considered. Problems involving collimated irradiation like the high temperature solar absorbers are usually dealt with by decomposing the radiative intensity into its collimated component $I_{c}$ and its diffuse component $I_{d}$ according to $I=I_{c}+I_{d}[2]$. The collimated intensity $I_{c}$ obeys the following equation (in stationary cases):

$$
\boldsymbol{u} \cdot \boldsymbol{\nabla} I_{c}=-\left(k_{a}+k_{s}\right) I_{c} \text { if } \boldsymbol{u}=\boldsymbol{u}_{\boldsymbol{c}}
$$

where $I_{c}$ is non-zero only in the collimated direction $\boldsymbol{u}_{\boldsymbol{c}}$ and can be written as:

$$
I_{c}(\boldsymbol{x}, \boldsymbol{u})=\delta\left(\boldsymbol{u}-\boldsymbol{u}_{\boldsymbol{c}}\right) I_{c}\left(\boldsymbol{x}_{\boldsymbol{w}}, \boldsymbol{u}\right) \exp \left(-\int_{0}^{\left\|\boldsymbol{x}-\boldsymbol{x}_{\boldsymbol{w}}\right\|}\left(k_{a}+k_{s}\right) d \ell\right),
$$

where $\delta$ is the Dirac distribution. The transport equation for $I_{d}$ is easily deduced by introducing the decomposition $I=I_{c}+I_{d}$ into the RTE,

$$
\boldsymbol{u} \cdot \nabla I_{d}=-\left(k_{a}+k_{s}\right) I_{d}+k_{a} I_{b}+k_{s} \int_{4 \pi} p\left(\boldsymbol{u} \cdot \boldsymbol{u}^{\prime}\right) I_{d}^{\prime} d \boldsymbol{u}^{\prime}+k_{s} p\left(\boldsymbol{u} \cdot \boldsymbol{u}_{\boldsymbol{c}}\right) I_{c}\left(\boldsymbol{x}, \boldsymbol{u}_{\boldsymbol{c}}\right),
$$

where $I_{d}^{\prime} \equiv I_{d}\left(\boldsymbol{x}, \boldsymbol{u}^{\prime}\right)$. Integrating Eq. 22 over the solid-angle leads to

$$
\nabla \cdot \boldsymbol{j}_{d}=k_{a}\left(4 \pi I_{b}-\rho_{d}\right)+k_{s} I_{c}\left(\boldsymbol{x}, \boldsymbol{u}_{\boldsymbol{c}}\right),
$$

where $\rho_{d}=\left\langle I_{d}\right\rangle$ and $\boldsymbol{j}_{d}=\left\langle I_{d} \boldsymbol{u}\right\rangle$. When the P1 approximation is assumed for the diffuse component $I_{d}=I_{d}^{\text {lim }}$, the diffusive flux $\boldsymbol{j}_{d}^{\text {lim }}=\left\langle I_{d}^{\text {lim }} \boldsymbol{u}\right\rangle$ can be written as:

$$
\boldsymbol{j}_{\boldsymbol{d}}^{\text {lim }}=-D \boldsymbol{\nabla} \rho_{d}^{l i m}+3 D k_{s} g I_{c}\left(\boldsymbol{x}, \boldsymbol{u}_{\boldsymbol{c}}\right) \boldsymbol{u}_{\boldsymbol{c}}
$$


The following equation is then obtained for the transport of $\rho_{d}^{\text {lim }}=\left\langle I_{d}^{\lim }\right\rangle$ :

$$
-\boldsymbol{\nabla} \cdot\left(D \nabla \rho_{d}^{\text {lim }}\right)=k_{a}\left(4 \pi I_{b}-\rho_{d}^{\text {lim }}\right)+k_{s} I_{c}\left(\boldsymbol{x}, \boldsymbol{u}_{\boldsymbol{c}}\right)-\boldsymbol{\nabla} \cdot\left[3 D k_{s} g I_{c}\left(\boldsymbol{x}, \boldsymbol{u}_{\boldsymbol{c}}\right) \boldsymbol{u}_{\boldsymbol{c}}\right] .
$$

In the HTD model, the decomposition of the radiative intensity becomes:

$$
I(\boldsymbol{x}, \boldsymbol{u})=I_{c}(\boldsymbol{x}, \boldsymbol{u})+\frac{\rho_{d}^{\text {lim }}(\boldsymbol{x})}{4 \pi}+\epsilon(\boldsymbol{x}, \boldsymbol{u}) .
$$

The transport equation for $\epsilon$ is the same as Eq. 11, only the source term differs and depends also on $I_{c}$ :

$$
S_{I_{c}, \rho^{l i m}}=k_{s} p\left(\boldsymbol{u} \cdot \boldsymbol{u}_{\boldsymbol{c}}\right) I_{c}\left(\boldsymbol{x}, \boldsymbol{u}_{\boldsymbol{c}}\right)+k_{a}\left(I_{b}-\frac{\rho_{d}^{\lim }}{4 \pi}\right)-\frac{\boldsymbol{u} \cdot \boldsymbol{\nabla} \rho_{d}^{\lim }}{4 \pi}
$$

The HTD model is then composed of a system of three equations (20-25-11).

\subsection{Monte Carlo algorithm for the HTD model}

The MCM has been chosen to simulate the mesoscopic equation of the HTD model in this example. The advantages of using the MCM in concentrating solar applications have been recently reviewed in [6]. In particular, it is preferred to other methods because of its ability to deal with geometrically complex systems. The modifications needed to take into account the collimated radiation in the $\mathrm{MC}$ algorithm presented in Appendix A are easily deduced from the integral formulation of the diffuse photon fluence rate $\rho_{d}(\boldsymbol{x})=\int_{4 \pi} I_{d}(\boldsymbol{x}, \boldsymbol{u}) d \boldsymbol{u}$, similar to the integral formula in Eq. 13:

$$
\rho_{d}(\boldsymbol{x})=\int_{4 \pi} p_{\boldsymbol{u}}(\boldsymbol{u}) d \boldsymbol{u} \int_{0}^{+\infty} p_{L}\left(\ell_{1}\right) d \ell_{1} \int_{4 \pi} p_{\boldsymbol{U}_{\mathbf{1}}}\left(\boldsymbol{u}_{\mathbf{1}}\right) d \boldsymbol{u}_{\mathbf{1}} \times w_{d}
$$

where $w_{d}$ is the Monte Carlo weight expressed by

$$
w_{d}=4 \pi\left\{H\left(\ell_{1}-d_{1}\right) I\left(\boldsymbol{x}_{\boldsymbol{w}}, \boldsymbol{u}\right)+H\left(d_{1}-\ell_{1}\right)\left\{\left(1-\omega\left(\boldsymbol{x}_{\mathbf{1}}\right)\right) I_{b}\left(\boldsymbol{x}_{\mathbf{1}}\right)+\omega\left(\boldsymbol{x}_{\mathbf{1}}\right) p\left(\boldsymbol{u} \mid \boldsymbol{u}_{\boldsymbol{c}}\right) I_{c}\left(\boldsymbol{x}_{\mathbf{1}}, \boldsymbol{u}_{\boldsymbol{c}}\right)+\omega\left(\boldsymbol{x}_{\mathbf{1}}\right) I_{d}\right.\right.
$$

Concerning the HTD model, the algorithm is almost identical to the one presented in section 2.3. The integrals are the same as given in Eqs. 18 and 19, the only difference being the term $S_{I_{c}, \rho^{l i m}}$ (Eq. 27) which replaces the term $S_{\rho^{l i m}}$ (Eq. 12).

\subsection{Results}

The HTD model is applied in this section to a test case related to a solar receiver and compared to the RTE. Since the resolution of the diffusion equation is very fast 
in this test-case, the CPU time for the RTE and for the HTD are almost the same $\left(t_{H T D}=1.01 t_{R T E}\right)$. The incident radiation $\rho(\boldsymbol{x})$ is computed inside a volumetric solar absorber considered as a porous medium having effective grey radiative properties. This porous medium is assumed one-dimensional at uniform temperature (1300K) and submitted to collimated irradiation $\left(800 \mathrm{~kW} / \mathrm{m}^{2}\right)$ at $x=0$ perpendicular to the slab. The following radiative properties were chosen, according to realistic values in solar applications $[10,11]$ : the slab optical thickness is $\tau=4$, the scattering albedo is $\omega=0.5$ and a diffuse sphere phase function [26] is chosen with an asymmetry factor $g=-4 / 9$. The simulation results for the isothermal solar receiver submitted to concentrated solar flux were obtained with $10^{5}$ bundles of rays for HTD and MCM.

Figures 3(a) and 3(b) present the results computed by the P1, HTD and RTE models, and the MC statistical errors, respectively. In figure 3(a), the P1, HTD and RTE solutions coincide for $x>0.5$, which means that the $\mathrm{P} 1$ model is the best one for radiative transfer in this zone of the receiver due to the lower computational requirements. However, for $x<0.5$, the P1 solution differs from the reference solution (RTE). In this application, the P1 approximation cannot be assumed close to the inlet $(x=0)$ boundary, where a maximum difference of $12 \%$ is observed between the P1 solution and the reference one.

The HTD solution is completely merged with the reference one, as expected. Figure 3(b) shows the small normalized statistical error, i.e., an uncertainty lower than $0.2 \%$ for a $99 \%$ confidence interval. The uncertainty for the HTD model is lower than the uncertainty for the RTE at equivalent computational time for a large part of the domain. Only close to the boundary $x=0$ where the solar irradiation enters the system, the RTE has a smaller relative error than the HTD solution. This is due to the error on the boundary condition of the P1 model at $x=0$. Consequently, the control variate approach is not useful anymore at this boundary.

In these applications, the HTD model can correct the prediction made by the P1 model close to the irradiated surface, and represents therefore an efficient alternative to the RTE or the P1 model. 


\section{Example of optical tomography application: transient radiative transfer with collimated irradiation}

\subsection{Test case}

The HTD model is now tested on the classical 1D test case related to optical tomography applications of a slab submitted to a short-pulse laser irradiation on one of its faces [27, 28]. The temporal pulse shape is a truncated Gaussian distribution. The boundary condition for the radiative intensity at point $x=0$ is

$$
I(0, \boldsymbol{u}, t)=I_{0} \delta\left(\boldsymbol{u}-\boldsymbol{u}_{\boldsymbol{c}}\right) \exp \left[-4 \ln 2\left(\frac{t-t_{c}}{t_{p}}\right)^{2}\right] \quad, \quad 0<t<2 t_{c},
$$

where $I_{0}$ is the maximum radiative intensity of the pulse which occurs at $t=t_{c}=3 t_{p}$. For $t>2 t_{c}$, the face is free from irradiation i.e. $I\left(0, \boldsymbol{u}, t>2 t_{c}\right)=0$. The medium inside the slab is cold (emission is neglected), absorbing and scattering. The HenyeyGreenstein phase function is used and the absorbing and scattering coefficients are homogeneous. The irradiation is a pulse normal to the left face of the slab $\left(\boldsymbol{u}_{\boldsymbol{c}} \cdot \boldsymbol{n}=1\right)$. The dimensionless time defined by $t_{p}^{*}=c\left(k_{a}+k_{s}\right) t_{p}$ is fixed to 0.5. The dimensionless transmittance $T(t)=\frac{1}{I_{0}} \int_{2 \pi} I(x=L, \boldsymbol{u}, t)(\boldsymbol{u} \cdot \boldsymbol{n}) d \boldsymbol{u}$ is estimated as:

$$
\begin{aligned}
T(t) & =T_{c}(t)+T_{d}(t) \\
& =\frac{1}{I_{0}} I_{c}\left(x=L, \boldsymbol{u}_{\boldsymbol{c}}, t\right)\left(\boldsymbol{u}_{\boldsymbol{c}} \cdot \boldsymbol{n}\right)+\frac{1}{I_{0}} \int_{2 \pi} I_{d}(x=L, \boldsymbol{u}, t)(\boldsymbol{u} \cdot \boldsymbol{n}) d \boldsymbol{u}
\end{aligned}
$$

where $L$ is the slab width $(x \in[0, L])$, and $\boldsymbol{n}$ is the outgoing normal at point $x=L$.

\subsection{Modified RTE and DE for transient radiative transfer with collimated irradiation}

In the transient regime, the transport equation for $I_{c}$ is written as:

$$
\frac{1}{c} \frac{\partial I_{c}}{\partial t}+\boldsymbol{u} \cdot \nabla I_{c}=-\left(k_{a}+k_{s}\right) I_{c}
$$

and for $I_{d}$ :

$$
\frac{1}{c} \frac{\partial I_{d}}{\partial t}+\boldsymbol{u} \cdot \nabla I_{d}=-\left(k_{a}+k_{s}\right) I_{d}+k_{a} I_{b}+k_{s} \int_{4 \pi} p\left(\boldsymbol{u} \cdot \boldsymbol{u}^{\prime}\right) I_{d}^{\prime} d \boldsymbol{u}^{\prime}+k_{s} p\left(\boldsymbol{u} \cdot \boldsymbol{u}_{\boldsymbol{c}}\right) I_{c}\left(\boldsymbol{x}, \boldsymbol{u}_{\boldsymbol{c}}\right)
$$

The expression of $I_{c}(\boldsymbol{x}, \boldsymbol{u}, t)$ remains similar to the expression of $I_{c}(\boldsymbol{x}, \boldsymbol{u})$ obtained in the stationary case (see Eq. 21):

$$
I_{c}(\boldsymbol{x}, \boldsymbol{u}, t)=\delta\left(\boldsymbol{u}-\boldsymbol{u}_{\boldsymbol{c}}\right) I_{c}\left(\boldsymbol{x}_{\boldsymbol{w}}, \boldsymbol{u}, t_{w}\right) \exp \left(-\int_{0}^{\left\|\boldsymbol{x}-\boldsymbol{x}_{\boldsymbol{w}}\right\|}\left(k_{a}+k_{s}\right) d \ell\right)
$$


where $t_{w}=t-\frac{\left\|\boldsymbol{x}-\boldsymbol{x}_{\boldsymbol{w}}\right\|}{c}$ and $\boldsymbol{x}_{\boldsymbol{w}}$ is the boundary point defined by the intersection between the line passing through point $\boldsymbol{x}$ in the direction $-\boldsymbol{u}$ and the boundary. If the P1 approximation is assumed for the diffusive contribution $I_{d}^{l i m}=\frac{1}{4 \pi} \rho_{d}^{l i m}+\frac{3}{4 \pi} \boldsymbol{j}_{\boldsymbol{d}}^{\text {lim }} \cdot \boldsymbol{u}$ as well as the diffusion approximation $\frac{\partial j_{d}^{l i m}}{\partial t}=0$, the following $\mathrm{DE}$ is obtained for $\rho_{d}^{\lim }=\left\langle I_{d}^{\lim }\right\rangle:$

$\frac{1}{c} \frac{\partial \rho_{d}^{l i m}}{\partial t}-\nabla \cdot\left(D \nabla \rho_{d}^{l i m}\right)=k_{a}\left(4 \pi I_{b}-\rho_{d}^{l i m}\right)+k_{s} I_{c}\left(\boldsymbol{x}, \boldsymbol{u}_{\boldsymbol{c}}\right)-\nabla \cdot\left[3 D k_{s} g I_{c}\left(\boldsymbol{x}, \boldsymbol{u}_{\boldsymbol{c}}\right) \boldsymbol{u}_{\boldsymbol{c}}\right]$

\subsection{Modified HTD Model for transient radiative transfer with collimated irradiation}

Using the following decomposition for the radiative intensity

$$
I(\boldsymbol{x}, \boldsymbol{u}, t)=I_{c}(\boldsymbol{x}, \boldsymbol{u}, t)+\frac{\rho_{d}^{\lim }(\boldsymbol{x}, t)}{4 \pi}+\epsilon_{d}(\boldsymbol{x}, \boldsymbol{u}, t),
$$

the transport equation for $\epsilon_{d}$ is deduced from Eqs. 33 and 35:

$$
\frac{1}{c} \frac{\partial \epsilon_{d}}{\partial t}+\boldsymbol{u} \cdot \nabla \epsilon_{d}=-\left(k_{a}+k_{s}\right) \epsilon_{d}+k_{s} \int_{4 \pi} p\left(\boldsymbol{u} \cdot \boldsymbol{u}^{\prime}\right) \epsilon_{d}^{\prime} d \boldsymbol{u}^{\prime}+S_{I_{c}, \rho^{l i m}}
$$

The source term $S_{I_{c}, \rho^{\text {lim }}}$ depends now also on $I_{c}$ :

$$
S_{I_{c}, \rho^{l i m}}=k_{s} I_{c}\left(\boldsymbol{x}, \boldsymbol{u}_{\boldsymbol{c}}, t\right)\left(p\left(\boldsymbol{u} \cdot \boldsymbol{u}_{\boldsymbol{c}}\right)-\frac{1}{4 \pi}\right)+\frac{1}{4 \pi}\left\{\boldsymbol{\nabla} \cdot \boldsymbol{j}_{\boldsymbol{d}}^{\text {lim }}-\boldsymbol{u} \cdot \boldsymbol{\nabla} \rho_{d}^{l i m}\right\}
$$

where $\boldsymbol{j}_{\boldsymbol{d}}^{\text {lim }}$ is given by Eq. 24 .

\subsection{Numerical methods}

The diffusion Eq. 35 is solved by a finite-volume method with a second-order central differencing, and the time discretization is assumed with a fully explicit scheme. The resolution of the diffusion equation is much more faster than the resolution of the mesoscopic equation of the HTD model, or than the resolution of the RTE, since this equation is macroscopic and does not involve angular integrations.

In section 4.5, the DOM is chosen for solving the modified RTE and the mesoscopic equation of the HTD model. A fully explicit time scheme is chosen for the discretization of the transient term of all the equations. The transport terms in the mesoscopic equations are spatially discretized with the step scheme. The angular discretization is carried out using the $S_{12}$ quadrature scheme, which computes 168 directions in the sphere. The macroscopic part (Eq. 35) of the HTD model is discretized using a second 
order finite volume method. The grid is uniform and the same for all equations, and the number of spatial grid points is $N_{x}=200$. The CFL number has been fixed to 0.5 : $\Delta t=0.5 \frac{\Delta x}{c}$.

Results obtained with the MCM are also presented in section 4.6. The MCM algorithm used to solve the RTE is the reverse MC algorithm already detailed in [29, 30]. It is a backward algorithm which estimate the radiation quantity at an instant $t$ which is fixed. The photon path is generated in a reverse way from the end of the optical path at time $t$ and position $x=L$, until the begining of the optical path at time $t_{0}=t-\frac{d}{c}$, where $d$ is the total length of the optical path inside the system. This algorithm is similar to the algorithms presented in the previous section, and corresponds to the following integral:

$$
\begin{aligned}
T_{d}(t) & =\frac{1}{I_{0}} \int_{2 \pi} I(x=L, \boldsymbol{u}, t)(\boldsymbol{u} \cdot \boldsymbol{n}) d \boldsymbol{u} \\
& =\int_{2 \pi} p_{\boldsymbol{U}}(\boldsymbol{u}) d \boldsymbol{u} \int_{0}^{+\infty} p_{L_{1}}\left(\ell_{1}\right) d \ell_{1} \int_{4 \pi} p_{\boldsymbol{U}_{\mathbf{1}}}\left(\boldsymbol{u}_{\mathbf{1}}\right) d \boldsymbol{u}_{\mathbf{1}} \times w_{d}
\end{aligned}
$$

where $\boldsymbol{n}$ is the outgoing normal at the face $x=L$. The pdf $p_{L_{i}}\left(\ell_{i}\right)$ and $p_{\boldsymbol{U}_{\boldsymbol{i}}}\left(\boldsymbol{u}_{\boldsymbol{i}}\right)$ are given by Eqs. 16 and 17, respectively. The pdf for the random generation of $\boldsymbol{u}$ is given by $p_{\boldsymbol{U}}(\boldsymbol{u})=\frac{\boldsymbol{u} \cdot \boldsymbol{n}}{\pi}$, and the MC weight is expressed as:

$$
w_{d}=\frac{\pi}{I_{0}} H\left(d_{1}-\ell_{1}\right) \exp \left[-\int_{0}^{\ell_{1}} k_{a} d \ell\right]\left\{I_{c}\left(x_{1}, \boldsymbol{u}_{\boldsymbol{c}}, t_{1}\right) p\left(\boldsymbol{u}_{\mathbf{1}} \mid \boldsymbol{u}_{\boldsymbol{c}}\right)+I_{d}\left(x_{1}, \boldsymbol{u}_{\mathbf{1}}, t_{1}\right)\right\}
$$

where $t_{1}=t-\frac{\ell_{1}}{c}, d_{1}=\frac{L}{\boldsymbol{u} \cdot \boldsymbol{n}}$ and $x_{1}=L-\boldsymbol{u} \cdot \boldsymbol{n} \ell_{1}$. The algorithm deduced from Eqs. 39 and 40 generates an optical path in the reverse way from the position $x=L$. At each new scattering position $x_{i}$ inside the slab, the collimated radiation $I_{c}\left(x_{i}, \boldsymbol{u}_{c}, t_{i}\right)$ (where $t_{i}=t-\frac{\sum_{j=1}^{i} \ell_{j}}{c}$ ) is calculated using Eq. 34 and added to the MC weight. The generation of the optical path stops when it leaves the system.

This algorithm is easily adapted to the HTD model following the procedure described in section 2.3. If we set $T_{d}(t)=\frac{\rho^{l i m}(L, t)}{4 I_{0}}+T_{\epsilon}(t)$, the MCM estimates $T_{\epsilon}$, which can be written as:

$$
\begin{aligned}
T_{\epsilon}(t) & =\frac{1}{I_{0}} \int_{2 \pi} \epsilon(x=L, \boldsymbol{u}, t)(\boldsymbol{u} \cdot \boldsymbol{n}) d \boldsymbol{u} \\
& =\int_{2 \pi} p_{\boldsymbol{U}}(\boldsymbol{u}) d \boldsymbol{u} \int_{0}^{+\infty} p_{L_{1}}\left(\ell_{1}\right) d \ell_{1} \int_{4 \pi} p_{\boldsymbol{U}_{\mathbf{1}}}\left(\boldsymbol{u}_{\mathbf{1}}\right) d \boldsymbol{u}_{\mathbf{1}} \times w_{\epsilon}
\end{aligned}
$$


where the MC weight is given by

$$
w_{\epsilon}=\frac{1}{I_{0}} H\left(\ell_{1}-d_{1}\right) \exp \left[-\int_{0}^{\ell_{1}} k_{a} d \ell\right]\left\{\frac{S_{I_{c}, \rho^{l i m}}\left(x_{1}, t_{1}\right)}{k_{s}}+H\left(d_{1}-\ell_{1}\right) \epsilon\left(x_{1}, \boldsymbol{u}_{1}, t_{1}\right)\right\} .
$$

The MC algorithm used to estimate $T_{\epsilon}(t)$ differs from the algorithm that estimates $T_{d}(t)$ only in the source term added to the MC weight (like in the previous sections).

\subsection{Results obtained with the DOM}

The DOM has been used to compare the efficiency of the RTE and the HTD formulations. The computational time required by the RTE and by the HTD models is almost identical $\left(t_{H T D} \simeq 1.02 t_{R T E}\right)$, since the time needed to solve the DE is very short in comparison with the time needed to solve the mesoscopic equations $\left(t_{D E} \simeq 0.005 t_{R T E}\right)$.

In figure 4 , the results of the estimated transmittance obtained with the different models are displayed for an optical thickness $\tau=10$, an albedo $\omega=0.5$, and an isotropic phase function. The $\mathrm{P} 1$ solution is strongly inaccurate because the transmittance is a quantity defined at the boundary of the domain, where the error on the DE resolution is significant. Concerning the others models, the HTD/DOM solution has the same level of accuracy as the RTE/DOM.

In figure 5(a), the optical thickness has been increased to 20. The HTD/DOM solution has a smaller error than the RTE/DOM for the albedo equal to 0.5. When the albedo is changed to 0.9 , like in figure $5(\mathrm{~b})$, the difference between the solutions obtained with the RTE/DOM and the HTD/DOM is larger. The poor performance of the RTE/DOM in this case is caused by the influence of the in-scattering term $k_{s} \int_{4 \pi} p\left(\boldsymbol{u} \cdot \boldsymbol{u}^{\prime}\right) I_{d}^{\prime} d \boldsymbol{u}^{\prime}$ in Eq. 33. When the scattering coefficient is high, the in-scattering term of the RTE dominates the others terms of the equation, and therefore the simulations are very sensitive to any error on this term. That's why the RTE/DOM at large optical thickness is difficult to handle. In the DOM/HTD model, the in-scattering term $k_{s} \int_{4 \pi} p\left(\boldsymbol{u} \cdot \boldsymbol{u}^{\prime}\right) \epsilon_{d}^{\prime} d \boldsymbol{u}^{\prime}$ is considered in the mesoscopic equation. It can be related to the total in-scattering term according to the following relation (using the decomposition of the radiative intensity in Eq. 36):

$$
k_{s} \int_{4 \pi} p\left(\boldsymbol{u} \cdot \boldsymbol{u}^{\prime}\right) I_{d}^{\prime} d \boldsymbol{u}^{\prime}=k_{s} \frac{\rho_{d}^{l i m}}{4 \pi}+k_{s} \int_{4 \pi} p\left(\boldsymbol{u} \cdot \boldsymbol{u}^{\prime}\right) I_{d}^{\prime} d \boldsymbol{u}^{\prime}
$$


When the scattering coefficient increases, the diffusion approximation yields better

accuracy inside the domain, and consequently $\frac{\rho_{d}^{l i m}}{4 \pi}$ gets closer to the solution $I_{d}$, while $\epsilon_{d}$ tends to 0 by definition. Therefore, at large optical thicknesses, $k_{s} \int_{4 \pi} p\left(\boldsymbol{u} \cdot \boldsymbol{u}^{\prime}\right) \epsilon_{d}^{\prime} d \boldsymbol{u}^{\prime}$ does not dominate the other terms of the equation like in the RTE/DOM, and the multi-scale model performs better than the RTE.

In figures 6, the optical thickness and the albedo are maintained at 20 and 0.9 , respectively, and the influence of the asymmetry factor is investigated. In the case of $g=0.5$ (see figure 6(a)), the HTD/DOM solution is again very close to the reference MC estimations, while the classical RTE/DOM fails. Note that the RTE/DOM could be improved by using a higher-order numerical scheme for the time and space discretization, but the computational cost would increase significantly. Therefore, the HTD model is still a very good alternative to the RTE when the DOM is used. In figure 6(b), the asymmetry factor has been increased to 0.9. In this case, the HTD model cannot correct the initial error of the classical DOM, which is inaccurate in forward peaked scattering media [31, 32]. In future work, the performance of the DOM when the asymmetry factor is close to 1 must be improved in order to compare the different models with DOM when scattering is forward-peaked.

\subsection{Results obtained with the MCM}

In this section, the performance of the HTD model is compared to the RTE when the MCM is applied. In figures 7 and 8, one symbol of the MCM curves represents one Monte Carlo calculation. In figure 7, the estimated transmittance $T$ and its uncertainty $\sigma_{T}$ are displayed for an optical thickness $\tau=10$, an albedo $\omega=0.5$, and an isotropic phase function. The transmittance estimated by the HTD model is equal to $T=T_{\epsilon}+$ $T_{I_{c}}+T_{\rho^{l i m}}$, where $T_{\epsilon}$ (given by Eq. 41 ) is estimated by MCM, $T_{I_{c}}=I_{c}\left(L, \boldsymbol{u}_{\boldsymbol{c}}, t\right)\left(\boldsymbol{u}_{\boldsymbol{c}} \cdot \boldsymbol{n}\right)$ is given analytically by Eq. 34 and $T_{\rho^{l i m}}=\frac{\rho^{l i m}}{4}$, is estimated by the DE (Eq. 35).

In figure $7(\mathrm{a})$, the transmittance estimated with the RTE and with the HTD model are merged as expected. The uncertainties of the estimations, given in figure $7(\mathrm{~b})$, show that the statistical errors are of the same order. The HTD model gives better results for a time $t>3.8 \times 10^{-9} s$, while at previous times, the statistical error is higher than that of the RTE. The total transmittance is null at the beginning, and consequently, the MCM estimates a negative $T_{\epsilon}$ in order to correct the error made by the $\mathrm{DE}$ for the 
estimation of $T_{\rho l i m}$. It is well-known that trying to estimate a very small quantity by the sum of two larger opposite values $\left(T_{\rho_{\text {lim }}}\right.$ and $\left.T_{\epsilon}\right)$ remains numerically more difficult than calculating directly the quantity. But as soon as $T_{\epsilon}$ becomes positive, the HTD model has a smaller error on the estimation of $T$.

In figure 8, the uncertainties of the MCM estimation are displayed for an optical thickness of $\tau=20$, and an albedo $\omega=0.9$. In figure 8(a), the phase function is isotropic. As already discussed in the previous section, the medium in this case is close to the diffusive regime, and consequently the HTD model becomes much more efficient than the RTE. The statistical uncertainties displayed in figure 8(a) confirm the results observed with the DOM at the same optical thickness, albedo and asymmetry factor.

In figure $8(\mathrm{~b})$, the phase function is strongly anisotropic which means that the P1 approximation is not valid anymore in a large part of the domain. In this case, the error of the HTD solution remains equivalent to the error of the RTE solution, and confirms that the HTD model works correctly, even in propagation media far from the diffusive regime. It is concluded that the HTD model can deal with the diffusive and the kinetic regimes, and offers consequently a good alternative to the RTE for the treatment of multi-scale problems where both regimes can be found.

\section{Conclusion}

A new multi-scale hybrid transport-diffusion model for radiative transfer calculations is proposed in this work. This model is based on a transport equation for a mesoscopic quantity that estimates the difference between the RTE and the DE solutions. The HTD model enables to recover the solution of the RTE by simply adding the solution of the mesoscopic part and the solution of the diffusion part. Results are presented for stationary and transient radiative transfer in academic example of solar concentrated and optical tomography applications.

It is shown that the proposed model allows to improve the efficiency of radiative transfer calculations when the propagation medium is close to the diffusive regime. Moreover, a simple procedure to couple the RTE and the DE can be developed using the HTD model, and avoid the difficult treatment of the interface between the mesoscopic and the macroscopic subdomains. Another advantage of the HTD model is that 
the mesoscopic equation is very similar to the RTE, and consequently the numerical methods (MCM, DOM or FVM) developed over the last decades for the RTE can be applied without any additional difficulties.

In future work, the HTD model will be used for the development of an efficient domain-decomposition method, in order to couple the macroscopic DE and the RTE in a multidimensional solar absorber where frequency-dependent spectrum will be considered. The objective is to improve the radiative transfer calculations in terms of trade-off between accuracy and computational requirements in order to study the coupling with the fluid dynamics in the absorber. The domain-decomposition method will consist in evaluating radiative transfer with the DE in all the domain, and completing this estimation with the HTD model in zone where the macroscopic model is inaccurate, namely close to the boundaries of the absorber. This would require less CPU time than using a method that solves the RTE in the whole domain, for an equivalent accuracy, and the usual difficulties of the interface treatment $[19,20]$ between the mesoscopic model and the macroscopic model in domain-decomposition methods are completely bypassed using the HTD model.

\section{Acknowledgments}

Financial support of FCT-Fundação para a Ciência e a Tecnologia within the framework of project PTDC/EMS-ENE/1028/2012 is acknowledged.

\section{A. Monte Carlo algorithm for the estimation of the photon fluence rate}

The MCM may be regarded as a numerical method for solving integrals $[6,33]$. Therefore, a MC algorithm can be deduced from an integral as shown in the following. The MC algorithm presented hereafter serves as a basis for the MC algorithm presented in section 2.3. The photon fluence rate $\rho$ at position $\boldsymbol{x}$ can be written according to the 
RTE in its integral form:

$$
\begin{aligned}
\rho(\boldsymbol{x})= & \int_{4 \pi} I(\boldsymbol{x}, \boldsymbol{u}) d \boldsymbol{u} \\
= & \int_{4 \pi} d \boldsymbol{u}\left\{I\left(\boldsymbol{x}_{\boldsymbol{w} \mathbf{1}}, \boldsymbol{u}\right) \exp \left[-\int_{0}^{d_{1}}\left(k_{a}+k_{s}\right) d \ell\right]+\int_{0}^{d_{1}} \exp \left[-\int_{0}^{\ell_{1}}\left(k_{a}+k_{s}\right) d \ell\right] d \ell_{1}\{\right. \\
& \left.\left.k_{a}\left(\boldsymbol{x}_{\mathbf{1}}\right) I_{b}\left(\boldsymbol{x}_{\mathbf{1}}\right)+k_{s}\left(\boldsymbol{x}_{\mathbf{1}}\right) \int_{4 \pi} p\left(\boldsymbol{u} \mid \boldsymbol{u}_{\mathbf{1}}\right) I\left(\boldsymbol{x}_{\mathbf{1}}, \boldsymbol{u}_{\mathbf{1}}\right) d \boldsymbol{u}_{\mathbf{1}}\right\}\right\}
\end{aligned}
$$

where $d_{1}=\left\|\boldsymbol{x}-\boldsymbol{x}_{\boldsymbol{w} \mathbf{1}}\right\|, \boldsymbol{x}_{\boldsymbol{w} \mathbf{1}}$ is located at the boundary of the domain, and $\boldsymbol{x}_{\mathbf{1}}$ is defined by $\boldsymbol{x}_{1}=\boldsymbol{x}-\ell_{1} \times \boldsymbol{u}$. The notations used to describe the equations and the algorithm are illustrated in figure 1. The integral in Eq. 44 is reformulated as:

$$
\begin{aligned}
\rho(\boldsymbol{x})= & \int_{4 \pi} d \boldsymbol{u} \int_{0}^{+\infty} \exp \left[-\int_{0}^{\ell_{1}}\left(k_{a}+k_{s}\right) d \ell\right] d \ell_{1}\left\{H\left(\ell_{1}-d_{1}\right) I\left(\boldsymbol{x}_{\boldsymbol{w} \mathbf{1}}, \boldsymbol{u}\right)+\right. \\
& \left.H\left(d_{1}-\ell_{1}\right)\left\{\left(1-\omega\left(\boldsymbol{x}_{\mathbf{1}}\right)\right) I_{b}\left(\boldsymbol{x}_{\mathbf{1}}\right)+\omega\left(\boldsymbol{x}_{\mathbf{1}}\right) \int_{4 \pi} p\left(\boldsymbol{u} \mid \boldsymbol{u}_{\mathbf{1}}\right) I\left(\boldsymbol{x}_{\mathbf{1}}, \boldsymbol{u}_{\mathbf{1}}\right) d \boldsymbol{u}_{\mathbf{1}}\right\}\right\} .
\end{aligned}
$$

The MC algorithm is deduced by first defining the set of pdfs (given by Eqs. 15, 16 and 17) used to generate randomly the scattering positions and directions, and then by introducing these pdfs in the integral. The following integral is obtained

$$
\rho(\boldsymbol{x})=\int_{4 \pi} p_{\boldsymbol{U}}(\boldsymbol{u}) d \boldsymbol{u} \int_{0}^{+\infty} p_{L}\left(\ell_{1}\right) d \ell_{1} \int_{4 \pi} p_{\boldsymbol{U}_{\mathbf{1}}}\left(\boldsymbol{u}_{\mathbf{1}}\right) d \boldsymbol{u}_{\mathbf{1}} \times w
$$

with the corresponding MC weight

$$
w=4 \pi\left\{H\left(\ell_{1}-d_{1}\right) I\left(\boldsymbol{x}_{\boldsymbol{w} \mathbf{1}}, \boldsymbol{u}\right)+H\left(d_{1}-\ell_{1}\right)\left\{\left(1-\omega\left(\boldsymbol{x}_{\mathbf{1}}\right)\right) I_{b}\left(\boldsymbol{x}_{\mathbf{1}}\right)+\omega I\left(\boldsymbol{x}_{\mathbf{1}}, \boldsymbol{u}_{\mathbf{1}}\right)\right\}\left(y_{j}\right)\right.
$$

Note that the expression of $w$ is recursive, the radiative intensity $I\left(\boldsymbol{x}_{\mathbf{1}}, \boldsymbol{u}_{\mathbf{1}}\right)$ can be developed similarly to $I(\boldsymbol{x}, \boldsymbol{u})$. The algorithm deduced from the integral in Eq. 45 is described hereafter:

- 1. $i=1$, a loop over the $N$ th optical path is started

- 2. A direction $\boldsymbol{u}$ is generated according to the pdf $p_{\boldsymbol{U}}(\boldsymbol{u})$. The factor $W$ is intialized to $W=4 \pi$, the MC weight to $w_{i}=0$, and $j=0$.

- 3. $j=j+1$. The optical path continues with the random generation of a path length $\ell_{j}$. A new scattering position is deduced $\boldsymbol{x}_{j}=\boldsymbol{x}_{j-1}-\boldsymbol{u}_{\boldsymbol{j - 1}} \ell_{j}$. 
- 4. Two scenarios are possible:

- 4.1 The position $\boldsymbol{x}_{\boldsymbol{j}}$ is inside the domain $\left(\ell_{j}<d_{j}\right.$ where $\left.d_{j}=\left\|\boldsymbol{x}_{\boldsymbol{w} \boldsymbol{j}}-\boldsymbol{x}_{\boldsymbol{j}-\mathbf{1}}\right\|\right)$. The MC weight takes into account the emission at $\boldsymbol{x}_{j}: w_{i}=w_{i}+W \times(1-$ $\left.\omega\left(\boldsymbol{x}_{\boldsymbol{j}}\right)\right) I_{b}\left(\boldsymbol{x}_{\boldsymbol{j}}\right)$. A scattered direction is generated according to the phase function $p\left(\boldsymbol{u}_{j} \mid \boldsymbol{u}_{j-1}\right)$. The factor $W$ is implemented by the albedo : $W=$ $W \times \omega\left(\boldsymbol{x}_{\boldsymbol{j}}\right)$.

Go to step 3 .

- 4.2 The position $\boldsymbol{x}_{\boldsymbol{j}}$ is outside the domain $\left(\ell_{j}>d_{j}\right) . w_{i}=w_{i}+W \times$ $I\left(\boldsymbol{x}_{\boldsymbol{w} \boldsymbol{j}}, \boldsymbol{u}_{\boldsymbol{j}-\mathbf{1}}\right)$.

Go to next step.

- 5. Two scenarios are possible:

- 5.1 If $i<N, i=i+1$, go to step 2 for generating a new optical path.

- 5.2 If $i=N, \rho$ is estimated by

$$
\tilde{\rho}=\frac{1}{N} \sum_{i=1}^{N} w_{i}
$$

and its uncertainty $\sigma_{\rho}$ by

$$
\tilde{\sigma}_{\rho}=\frac{1}{\sqrt{N-1}} \sqrt{\frac{1}{N} \sum_{i=1}^{N} w_{i}^{2}-\left(\frac{1}{N} \sum_{i=1}^{N} w_{i}\right)^{2}} .
$$

End of the algorithm

[1] B. Davison, Neutron Transport Theory, Clarendon Press, Oxford, 1958.

[2] M. Modest, Radiative heat transfer, New-York: McGraw-Hill, 2003.

[3] A. Welch, M. van Gemert (Eds.), Optical-thermal response of laser-irradiated tissue, Plenum, New York, 1995.

[4] R. Goody, Y. Yung, Atmospheric radiation, Oxford university Press, 1989.

[5] J. Howell, The Monte Carlo method in radiative heat transfer, J. Heat Transf. 120 (3) (1998) 547-560. 
[6] J. Delatorre, et al., Monte Carlo advances and concentrated solar applications, Solar Energy http://dx.doi.org/10.1016/j.solener.2013.02.035.

[7] G. Bal, A. Davies, I. Langmore, A hybrid (Monte Carlo/deterministic) approach for multi-dimensional radiation transport, J. Comput. Phys. 230 (2011) 7723-7735.

[8] M. Roger, S. Blanco, M. E. Hafi, R. Fournier, Monte Carlo estimates of domaindeformation sensitivities, Physical Review Letter 95 (18).

[9] A. Avila-Marin, Volumetric receivers in solar thermal power plants with central receiver system technology: A review, Solar Energy 85 (5) (2011) 891 - 910.

[10] Z. Wu, C. Caliot, G. Flamant, Z. Wang, Coupled radiation and flow modeling in ceramic foam volumetric solar air receivers, Solar Energy 85 (9) (2011) 2374 2385.

[11] Z. Wu, Z. Wang, Fully coupled transient modeling of ceramic foam volumetric solar air receiver, Solar Energy 89 (2013) 122 - 133.

[12] S. Kumar, K. Mitra, Microscale aspects of thermal radiation transport and laser application, Adv Heat Transfer 33 (1999) 187-294.

[13] S. Wan, Z. Guo, S. Kumar, J. Aber, B. Garetz, Noninvasive detection of inhomogeneities in turbid media with time-resolved log-slope analysis, J. Quant. Spectrosc. Radiat. Transf. 84 (2004) 493-500.

[14] Z. Guo, S. Wan, K. Kim, C. Kosaraju, Comparing diffusion approximation with radiation transfer analysis for light transport in tissues, Optical Review 10 (5) (2003) 415-421.

[15] O. Lehtikangas, T. Tarvainen, Hybrid forward-peaked-scattering-diffusion approximations for light propagation in turbid media with low-scattering regions, J. Quant. Spectrosc. Radiat. Transf. 116 (2013) 132-144.

[16] L. Wang, S. Jacques, Hybrid model of monte carlo simulation and diffusion theory for light reflectance by turbid media, J. Opt. Soc. Am. A 10 (8) (1993) 1746-1752. 
[17] T. Tarvainen, M. Vauhkonen, V. Kolehmainen, S. Arridge, J. Kaipio, Coupled radiative transfer equation and diffusion approximation model for photon migration in turbid medium with low-scattering and non-scattering regions, Phys. Med. Biol. 50 (2005) 4913-4930.

[18] D. Gorpas, D. Yova, K. Politopoulos, A three-dimensional finite elements approach for the coupled radiative transfer equation and diffusion approximation modeling in fluorescence imaging, J. Quant. Spectrosc. Radiat. Transf. 111 (2010) 553-568.

[19] P. Degond, S. Jin, A smooth transition model between kinetic and diffusion equations, SIAM J. Numeric. Anal. 42 (2005) 2671-2687.

[20] M. Roger, N. Crouseilles, A dynamic multi-scale model for transient radiative transfer calculations, J. Quant. Spectrosc. Radiat. Transf. 116 (2013) 110-121.

[21] N. Crouseilles, P. Degond, M. Lemou, A hybrid kinetic-fluid model for solving the vlasov-BGK equation, J. Comput. Phys. 203 (2005) 572-601.

[22] M. Lemou, L. Mieussens, A new asymptotic preserving scheme based on micromacro formulation for linear kinetic equations in the diffusion limit, SIAM J. Sci. Comput. 31 (1) (2008) 334-368.

[23] P. Degond, G. Dimarco, L. Mieussens, A multiscale kinetic-fluid solver with dynamic localization of kinetic effects, J. Comput. Phys. 229 (2010) 4907-4933.

[24] M. Lemou, F. Méhats, Micro-macro schemes for kinetic equations including boundary layers, SIAM J. Sci. Comput. 34 (6) (2012) B734-B760.

[25] W. Star, Optical-thermal response of laser-irradiated tissue, chapter 6 : Diffusion Theory of Light Transport, Plenum, New York, 1995.

[26] R. Coquard, D. Baillis, J. Randrianalisoa, Homogeneous phase and multi-phase approaches for modeling radiative transfer in foams, International Journal of Thermal Sciences 50 (9) (2011) 1648 - 1663.

[27] J. Chai, Finite volume method for transient radiative transfer, Numerical Heat Transf., Part B 44 (2003) 187-208. 
[28] L. Ruan, S. Wang, H. Qi, D. Wang, Analysis of the characteristics of time-resolved signals for transient radiative transfer in scattering participating media, J. Quant. Spectrosc. Radiat. Transf. 111 (2010) 2405-2414.

[29] X. Lu, P. f. Hsu, Reverse Monte Carlo simulations of light pulse propagation in nonhomogeneous media, J. Quant. Spectrosc. Radiat. Transf. 93 (2005) 349-367.

[30] M. Roger, Modèles de sensibilité dans le cadre de la méthode de Monte-Carlo : Illustrations en transfert radiatif, Ph.D. thesis, INP Toulouse, France (2006).

URL http://ethesis.inp-toulouse.fr/archive/00000317/

[31] L. Liu, L. Ruan, H. Tan, On the discrete ordinates method for radiative heat transfer in anisotropically scattering media, Int. J. Heat Mass Transfer 45 (2002) 3259-3262.

[32] B. Hunter, Z. Guo, Conservation of asymmetry factor in phase function discretization for radiative transfer analysis in anisotropic scattering media, Int. J. Heat Mass Transfer 55 (2012) 1544-1552.

[33] J. Hammersley, D. Handscomb, Monte Carlo Methods, Chapman and Hall, London, 1964. 


\begin{tabular}{l|llll} 
& $\tau=1$ & $\tau=5$ & $\tau=10$ & $\tau=20$ \\
\hline$\frac{t_{H T D}}{t_{R T E}}$ & 1.3 & 0.85 & 0.6 & 0.35 \\
\hline
\end{tabular}

Table 1: Ratio of the CPU times needed for solving the HTD and the RTE models for different optical thicknesses. The albedo is $0.5\left(k_{a}=k_{s}\right)$ and the phase function is isotropic. 


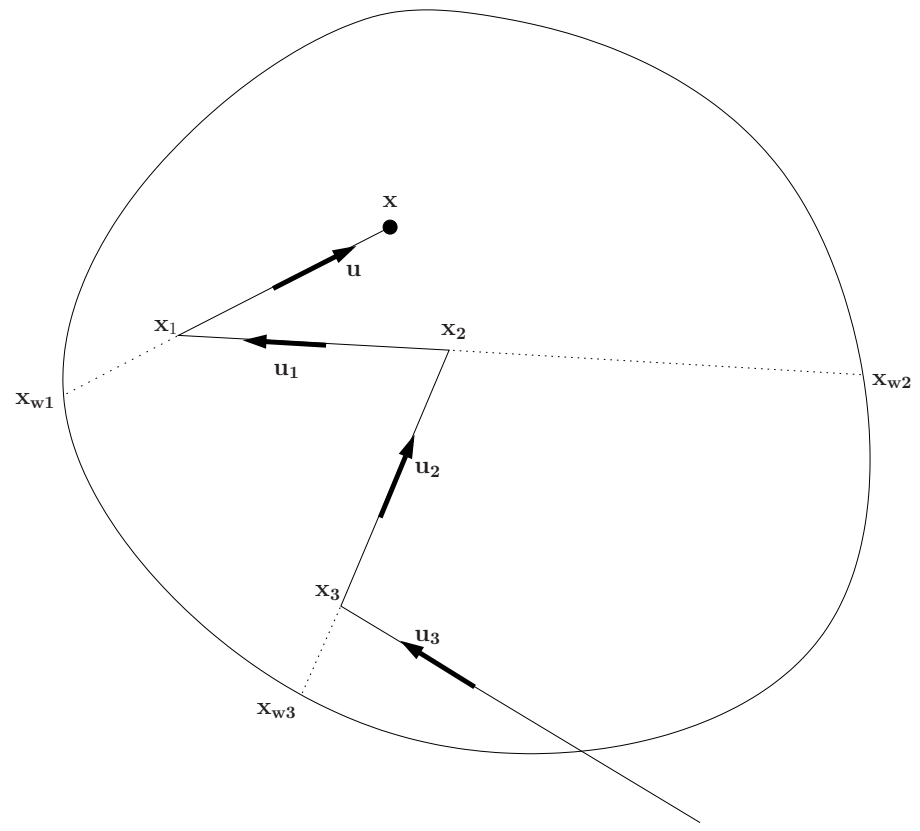

Figure 1: Illustration of the notations used in the integrals related to the MC algorithms with an example of a multiple scattering optical path. 


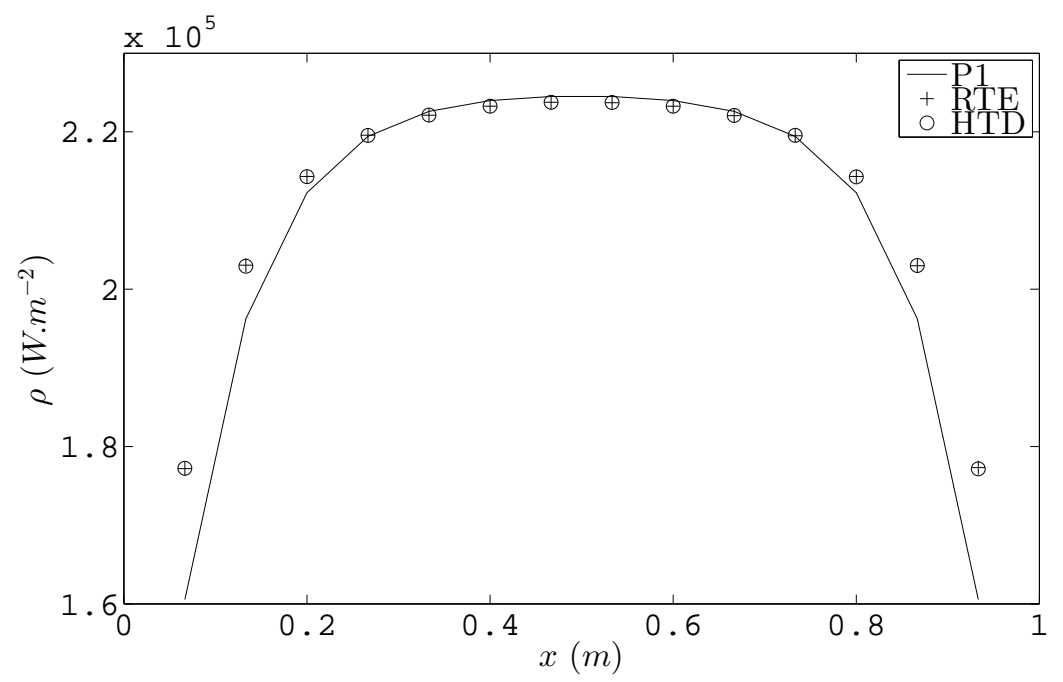

(a)

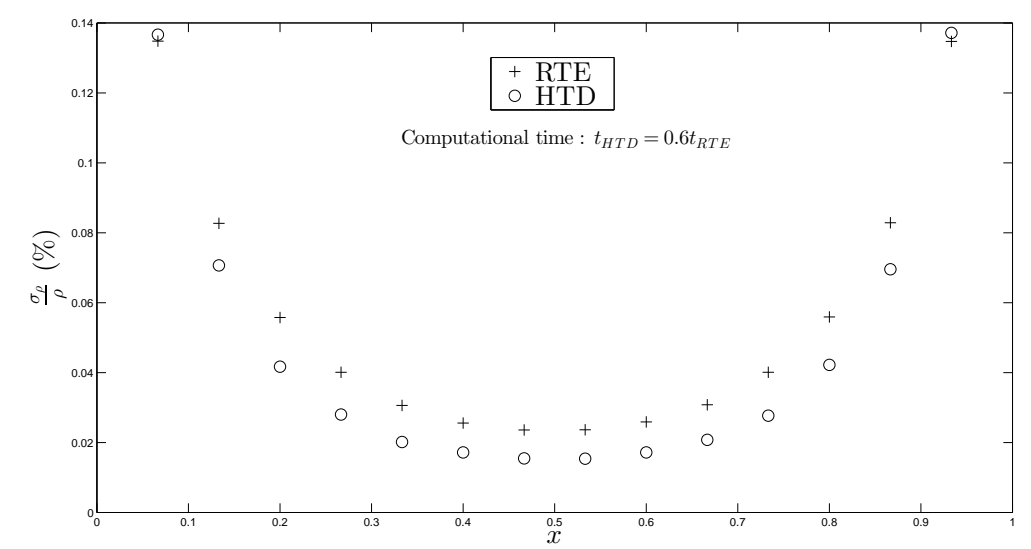

(b)

Figure 2: (a) Estimation of the photon fluence rate $\rho(x, y=0.6, z=0.6)$ in the cubic domain. MCM solutions of the RTE and the HTD model, and P1 solution. The optical thickness is $\tau=10$, the albedo $\omega=0.5$ and the cubic side is equal to $1 \mathrm{~m}$. (b) Statistical error related to the RTE and HTD simulations. 


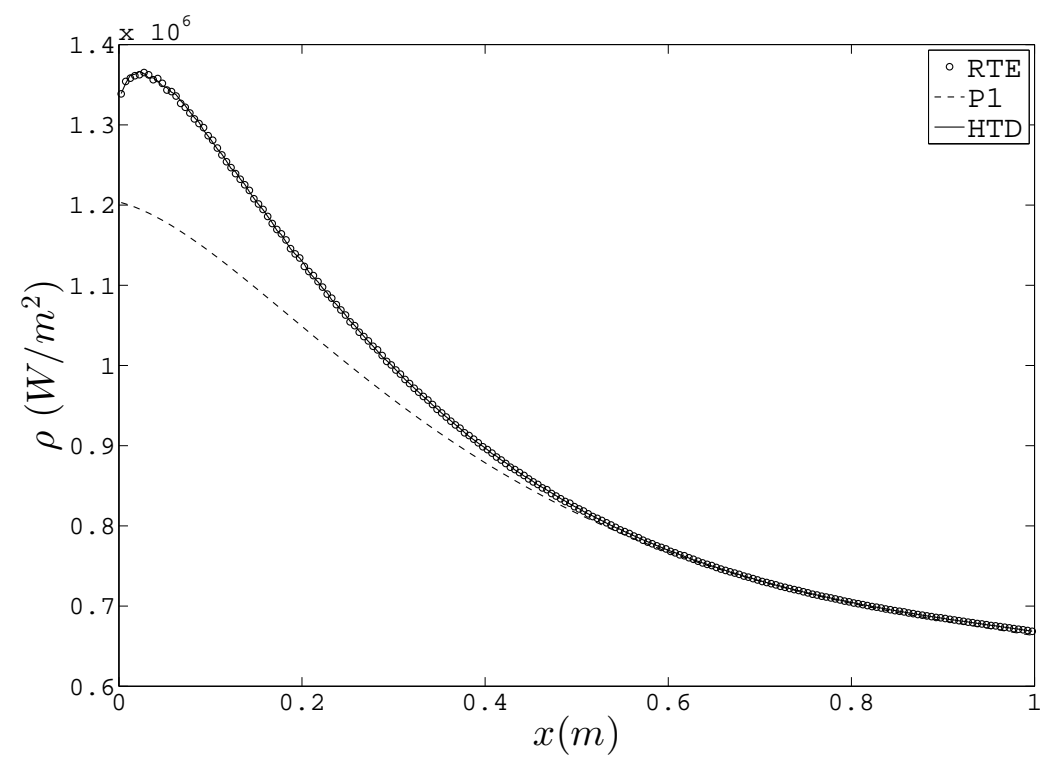

(a)

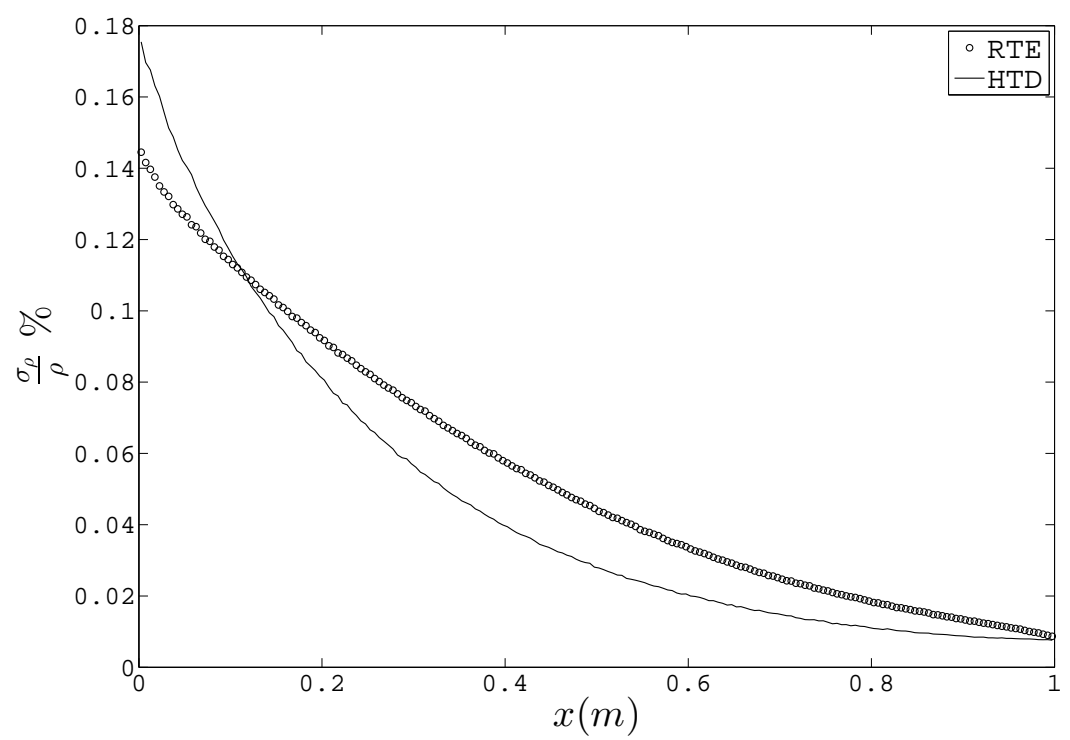

(b)

Figure 3: Irradiation profile inside the 1D solar absorber: (a) comparison between the RTE model estimated with MCM (reference solution), P1 and HTD models for an optical thickness $\tau=k_{a}+k_{s}=4$, an albedo $\omega=0.5$ and a diffuse sphere phase function. (b) Statistical error related to RTE and HTD simulations 


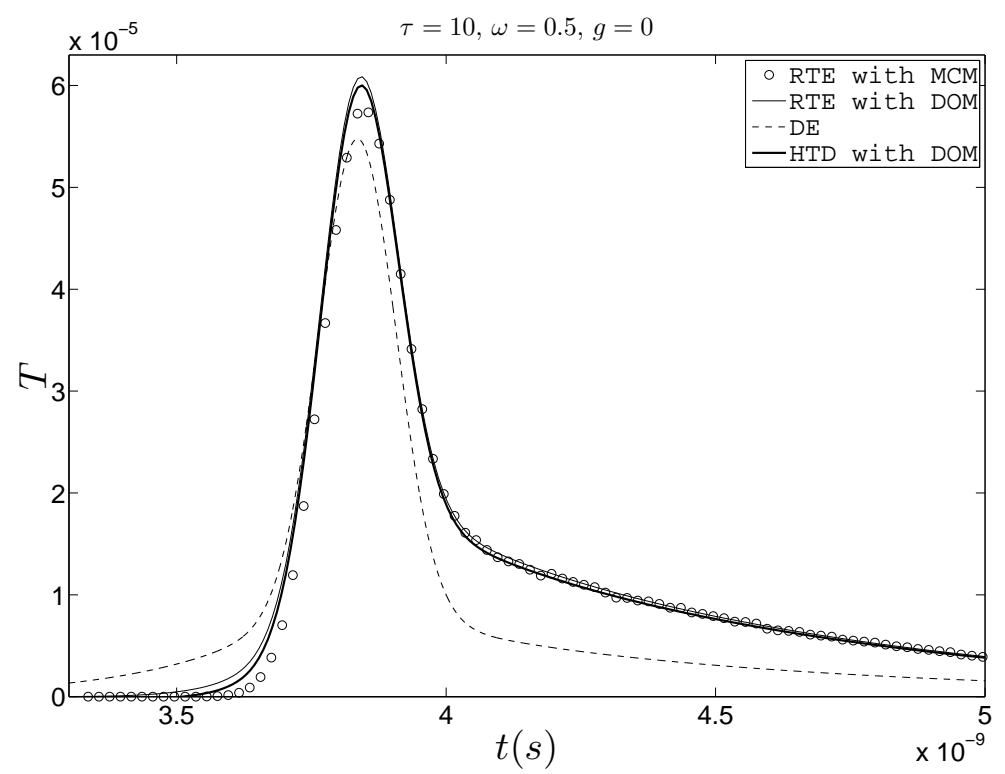

Figure 4: Time history of the dimensionless transmittance: comparison between the RTE solution estimated with MCM (reference solution), the RTE with DOM, the DE and the HTD models for an optical thickness $\tau=k_{a}+k_{s}=10$, an albedo $\omega=0.5$, and an isotropic phase function. 


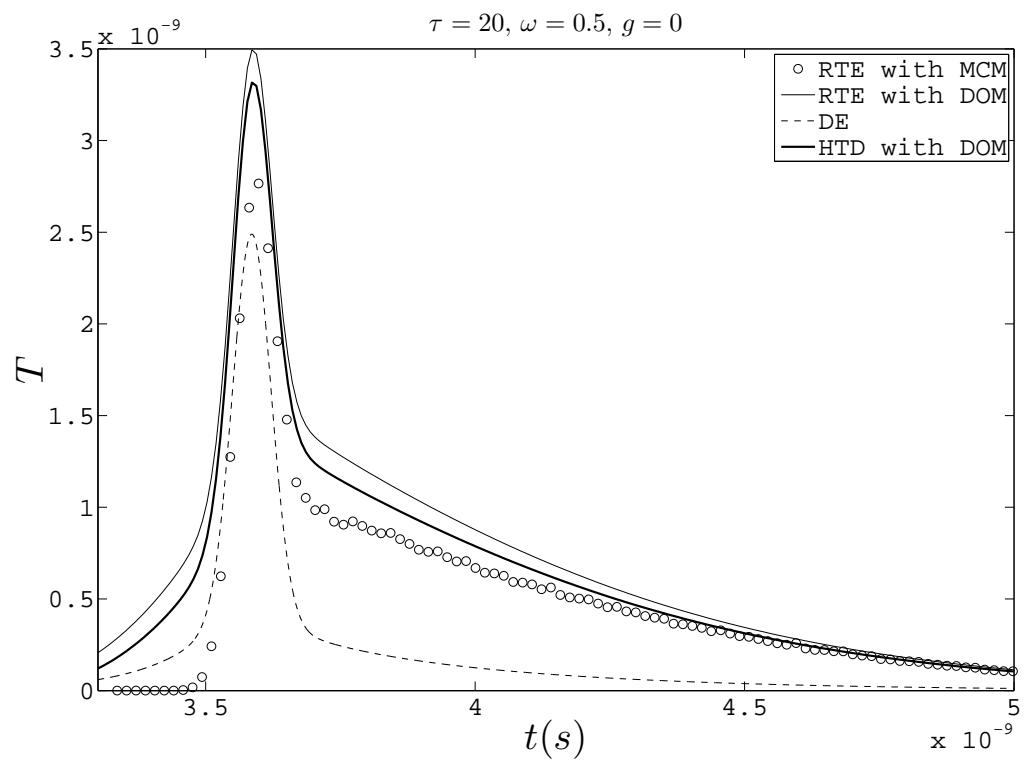

(a)

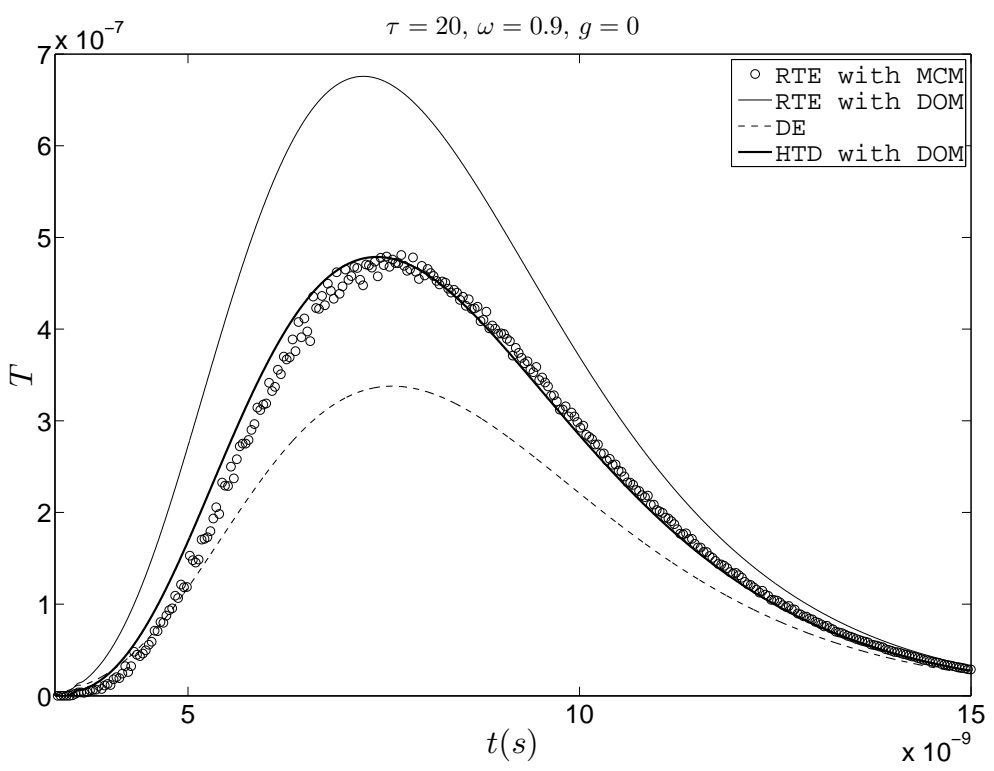

(b)

Figure 5: Time history of the dimensionless transmittance: comparison between the RTE solution estimated with MCM (reference solution), the RTE with DOM, the DE and the HTD models for an optical thickness $\tau=k_{a}+k_{s}=20$, an albedo $\omega=0.5$ (a) and 0.9 (b), and an isotropic phase function. 


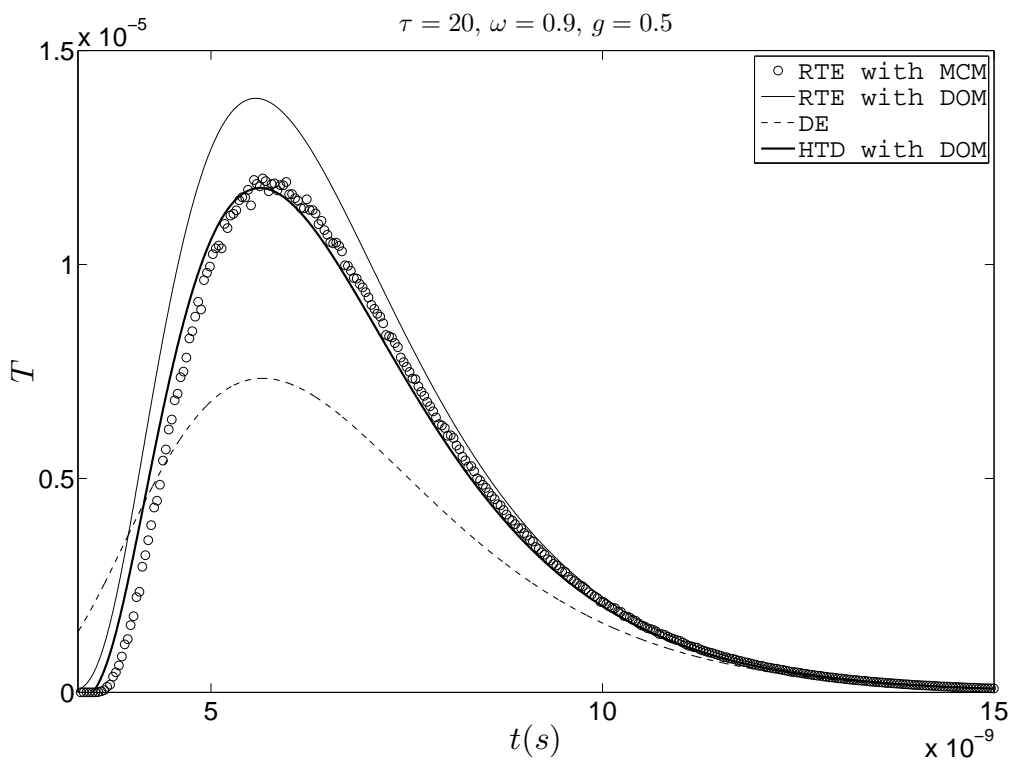

(a)

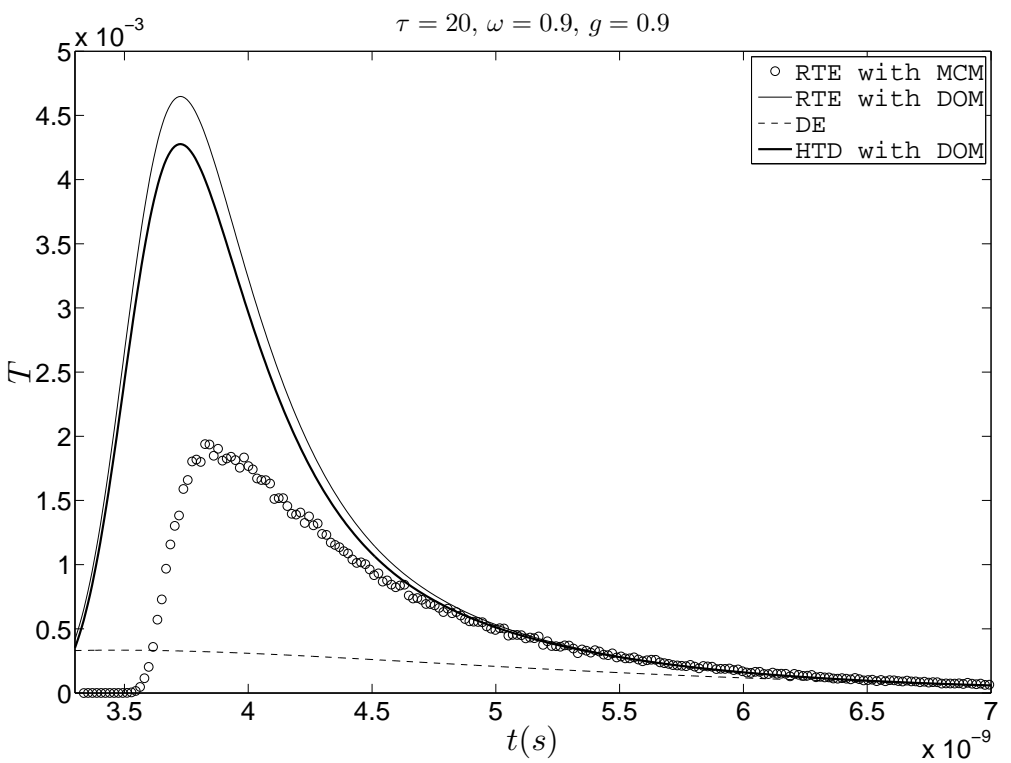

(b)

Figure 6: Time history of the dimensionless transmittance: comparison between the RTE model estimated with MCM (reference solution), the RTE with DOM, the DE and the HTD models for an optical thickness $\tau=k_{a}+k_{s}=20$, an albedo $\omega=0.9$, and a Henyey-Greenstein phase function with $g=0.5$ (a) and with $g=0.9$ (b). 


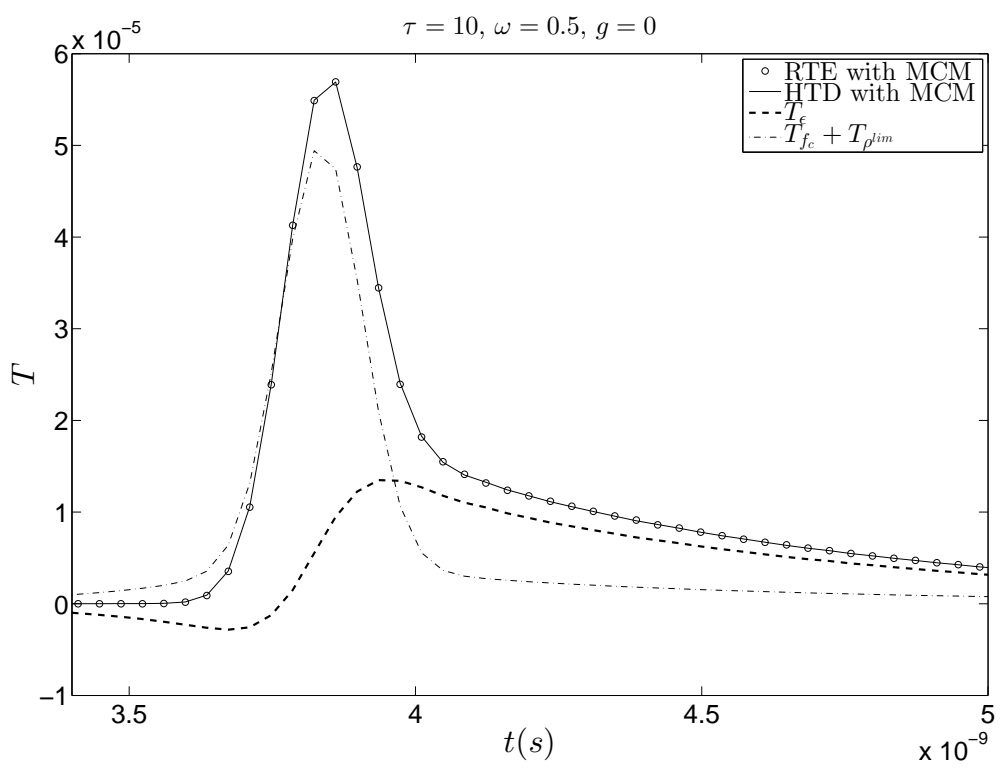

(a)

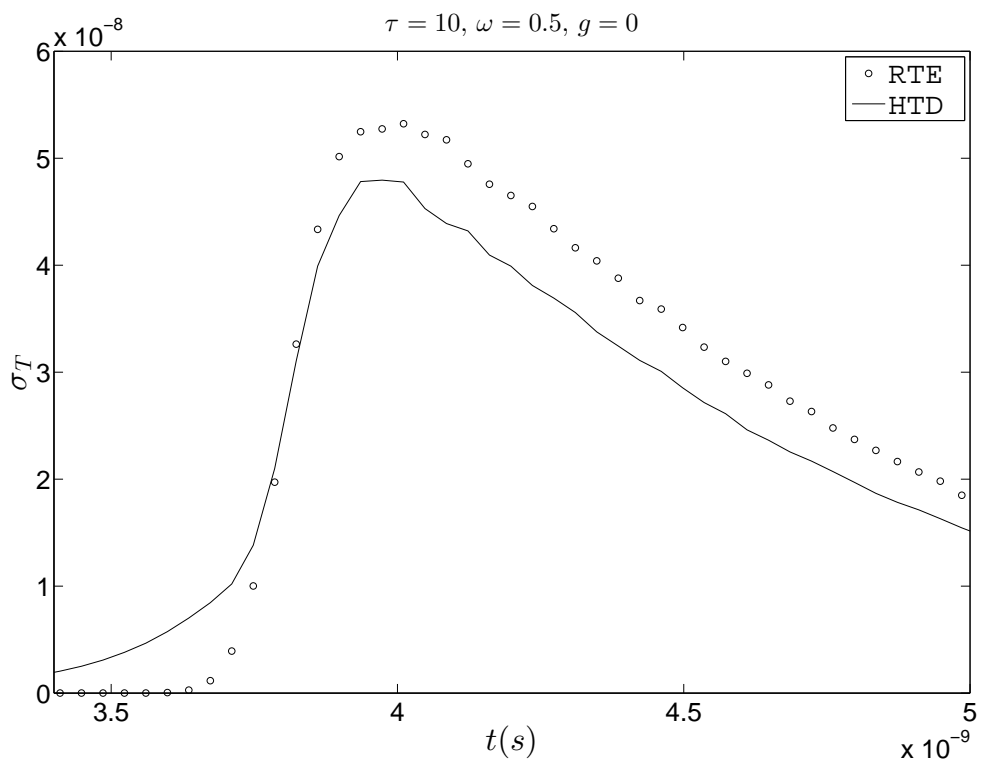

(b)

Figure 7: MCM solutions of the RTE and the HTD models. Time history of the dimensionless transmittance (a), and of the statistical uncertainties (b) for an optical thickness $\tau=k_{a}+k_{s}=10$, an albedo $\omega=0.5$, and an isotropic phase function. 


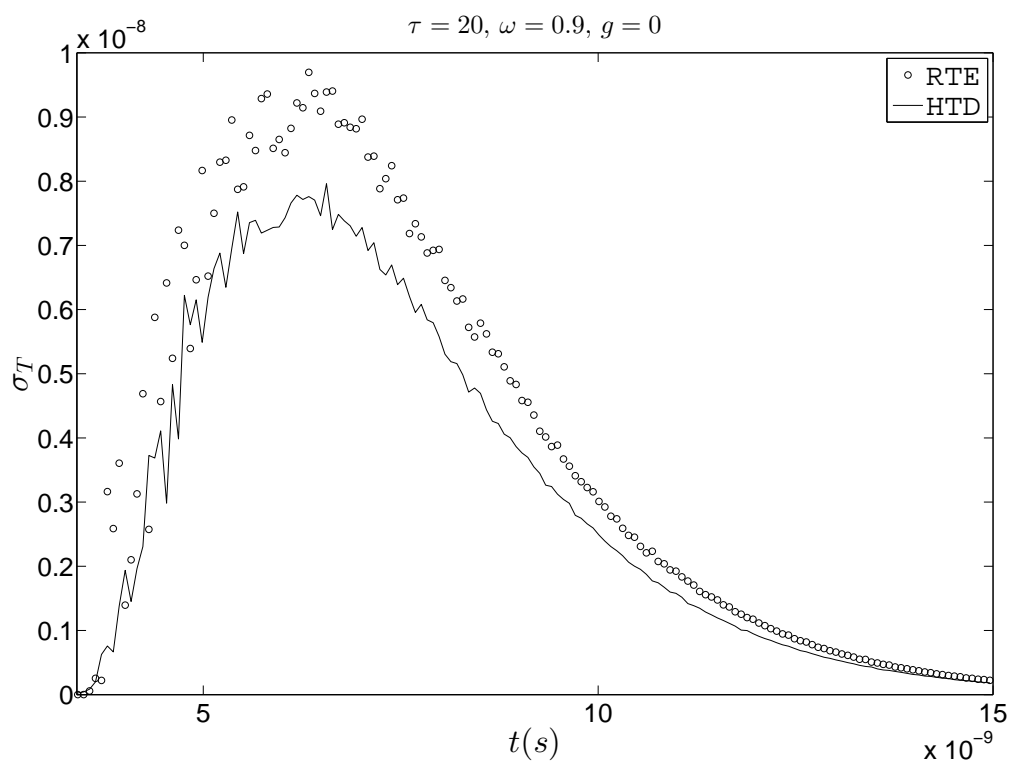

(a)

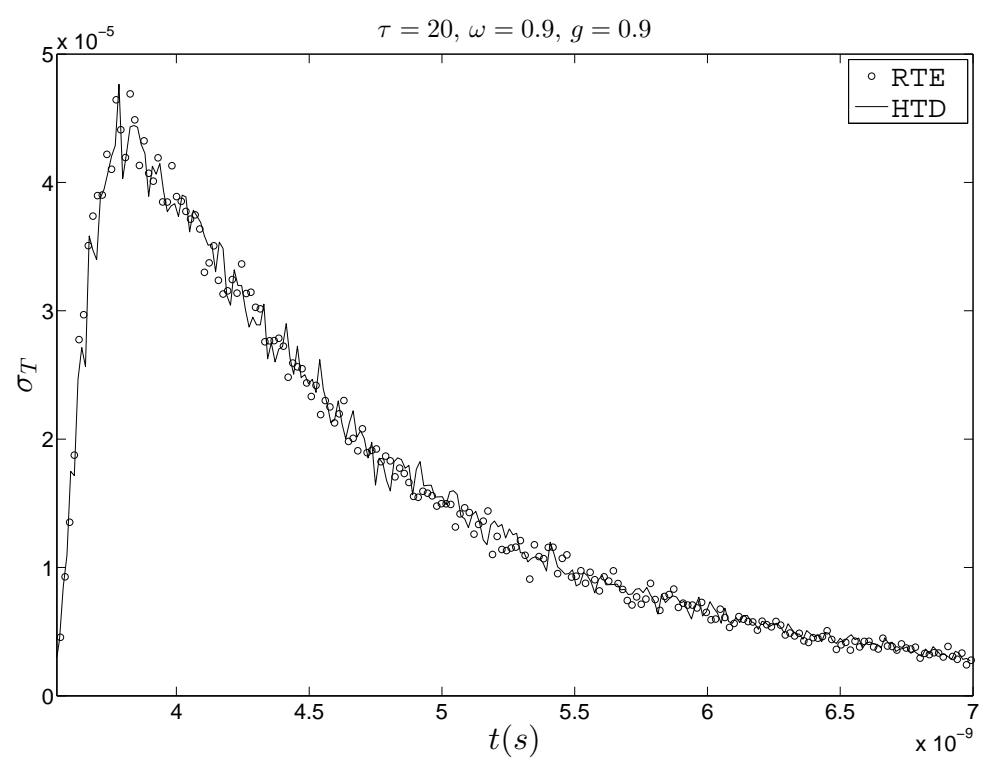

(b)

Figure 8: Statistical uncertainties of the MCM solutions obtained by the RTE and by the HTD model for an optical thickness $\tau=k_{a}+k_{s}=20$, an albedo $\omega=0.9$, and an isotropic phase function (a) or a Henyey-Greenstein phase function with $g=0.9$ (b). 